\title{
Synthesis of 2-aroyl-(4 or 5)-aryl-1H-imidazoles and 2-hydroxy-3,6-diaryl-pyrazines via a cascade process
}

\author{
Cong Liu, Rong J. Dai,* Guo W. Yao, and Yu L. Deng \\ School of Life Science, Beijing Institute of Technology, Beijing 100081, China \\ E-mail: dairongji@bit.edu.cn
}

DOI: http://dx.doi.org/10.3998/ark.5550190.p008.270

\begin{abstract}
The synthesis of (4 or 5)-aryl-2-aroyl-1H-imidazoles and 2-hydroxy-3,6-diarylpyrazines from aryl methyl ketones via a cascade process of DMSO-HBr oxidation and Debus reaction was investigated. Owing to the simple starting materials, mild conditions, easy operation, high bioactivity of imidazole and pyrazine derivatives, this protocol has great potential in medicinal chemistry.
\end{abstract}

Keywords: Debus-Radziszewski condensation, cascade reactions, imidazole synthesis, DMSO-HBr oxidation, pyrazine synthesis

\section{Introduction}

Imidazole moiety exists widely in biological products and important chemical blocks, such as essential amino acid histidine, hormone histamine, antifungal drug nitroimidazoles, the sedative midazolam and so on. ${ }^{1,2}$ Among the big family of imidazole derivatives, (4 or 5)aryl-2-aryloyl-(1H)-imidazoles (AAIs) exhibit many special properties. For example, topsentin (Figure 1) is a bis-indole alkaloid isolated from the Mediterranean sponge Topsentia genitrix. ${ }^{3}$ Topsentin derivatives have been detected to have antitumor and antiviral activities. ${ }^{4}$ The alkaloid 2-(p-hydroxybenzoyl)-4-( $p$-hydroxy-phenyl) imidazole (Figure 1) is a nature product from marine organism, which performs well in inhibiting human aldose reductase. ${ }^{5}(4$ or 5)-Furan-2-yl-2-furoyl-1H-imidazole (FFI) is a major fluorescent advanced end product of proteins exposed to glucose over long periods. ${ }^{6-8}$ The determination of FFI can be used to measure protein aging. 9,10 AAIs also can be used as starting materials to synthesize more complicated bioactive compounds like imidazo-[1,2- $a$ ]pyridine moieties, which have been shown to possess diverse therapeutic activities. ${ }^{11}$ In recent years, AAI derivatives have been the subject of biological and chemical research. ${ }^{12}$ 

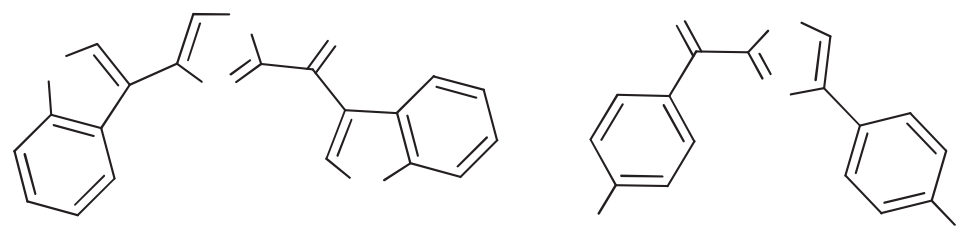

Figure 1. Structures of topsentin A and 2-( $p$-hydroxybenzoyl)-4-( $p$-hydroxyphenyl)imidazole.

Many syntheses have been reported since the imidazole core was first synthesized by Debus in 1858. ${ }^{13-16}$ Now the Debus-Radziszewski condensation is still used for creating Csubstituted imidazoles. Cascade reaction is a useful procedure and widely employed for synthesis of heterocyclic compounds. ${ }^{17-20}$ Cascade process not only reduces the costs for waste management, energy supplies, and materials, but also helps to save natural resources. In this paper, commercial acetyl aromatic compounds were used as the substrates. After a cascade process of DMSO-HBr oxidation and Debus-Radziszewski condensation, (4 or 5)aryl-2-aryloyl-(1H)-imidazoles and 2-hydroxy-3,6-diaryl-pyrazines could be deposited separately from the solvents. Compared with the previously reported method of synthesizing AAIs, ${ }^{28}$ this route is of characteristic of low cost, less pollution and easy operation.

\section{Results and Discussion}

Selenium dioxide is a common oxidant to synthesize phenyl glyoxal. However, $\mathrm{Se}$ and $\mathrm{SeO}_{2}$ are hypertoxic substances with high biological toxicity to aquatic organisms. ${ }^{21-23}$ According to the literature, DMSO-HBr system could give a good yield in oxidizing acetophenone. ${ }^{24}$ It is a mild, easy operating process with low toxicity. ${ }^{25,26}$ The original plan of our research was employing the cascade process of DMSO oxidation and Debus reaction to synthesize 4-(3pyridyl)-(1H)-imidazole, the key intermediate for preparing telithromycin (Scheme 1, Route 1). ${ }^{27}$ Accidentally, we indentified the product to be (4 or 5)-(3-pyridyl)-2-(3-pyridinoyl)-(1H)imidazole when only ammonia other than the mixture of ammonia and formaldehyde was used to trigger Debus reaction (Scheme 1, Route 2). Furthermore, the product could precipitate from the solution with high purity.

Considering the high bioactivity of AAI derivatives, a more detailed research was carried out on this procedure utilizing acetophenone to be the substrate. As the conditions of DMSO$\mathrm{HBr}$ oxidation have been confirmed, ${ }^{27}$ our investigations were focused on the favorable conditions of Debus condensation in the presence of various amines (Table 1). 


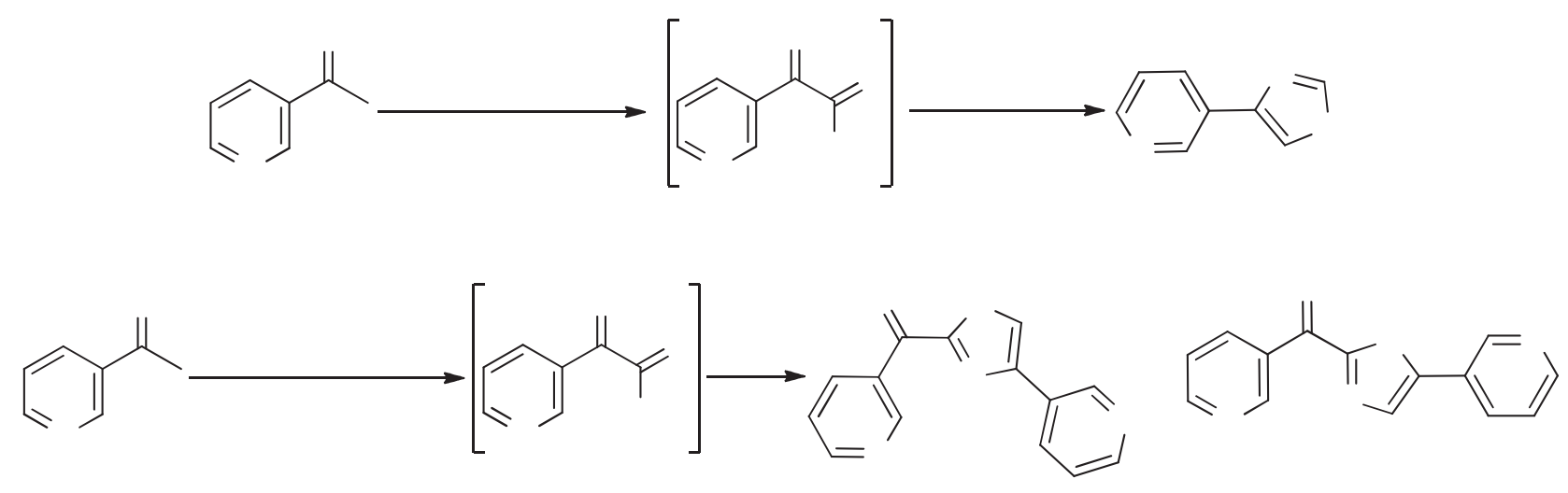

Scheme 1. The cascade DMSO-HBr oxidation and Debus-Radziszewski condensation procedure.

Table 1. Optimization of the reaction conditions

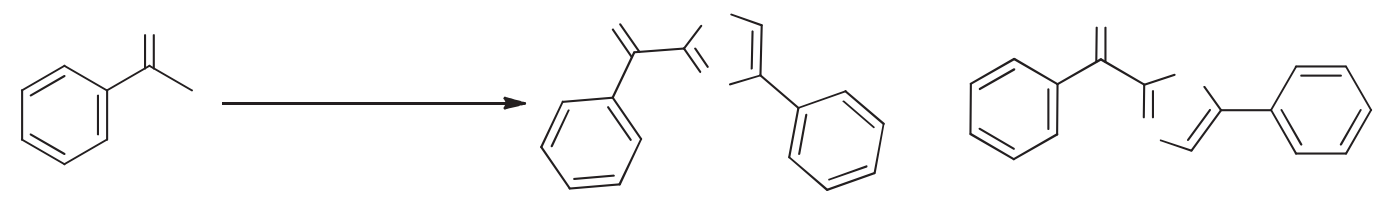

\begin{tabular}{cccc}
\hline Entry & Amine & $\mathrm{T}\left({ }^{\circ} \mathrm{C}\right)$ & Yield $^{\mathrm{a}}(\%)$ \\
\hline 1 & $\mathrm{NH}_{3} \cdot \mathrm{H}_{2} \mathrm{O}$ & 20 & 80 \\
2 & $\mathrm{NH}_{4} \mathrm{Cl}$ & 20 & 0 \\
3 & $\mathrm{NH}_{4} \mathrm{HCO}_{3}$ & 20 & 59 \\
4 & $\left(\mathrm{NH}_{4}\right)_{2} \mathrm{CO}_{3}$ & 20 & 61 \\
5 & $\mathrm{CH}_{3} \mathrm{COONH}_{4}$ & 20 & 65 \\
6 & $\mathrm{Urotropine}_{7}$ & 20 & 0 \\
7 & $\mathrm{NH}_{3} \cdot \mathbf{H}_{2} \mathrm{O}$ & $\mathbf{0 - 5}$ & $\mathbf{8 3}$ \\
8 & $\mathrm{NH}_{3} \cdot \mathrm{H}_{2} \mathrm{O}$ & 40 & 79 \\
9 & $\mathrm{NH}_{3} \cdot \mathrm{H}_{2} \mathrm{O}$ & 60 & 77 \\
10 & $\mathrm{NH}_{3} \cdot \mathrm{H}_{2} \mathrm{O}$ & 80 & 73 \\
\hline
\end{tabular}

${ }^{\text {a }}$ Isolated yields.

According to the literature, the product of $\mathbf{1 a}$ had two isomeric 2-aryloylimidazoles $\mathbf{2 a}$ and $2 \mathbf{a}^{\prime}{ }^{28}$ So in this paper, all yields of AAIs referred to the yields of the two isomers. Most 
ammonium salts were less active than aqueous ammonia in this process (Table 1, entries 1-7). So aqueous ammonia became the best choice. A higher conversion rate was observed when the reaction was carried out under low temperature (Table 1, entries 7-10). Ultimately, optimal conditions were identified, that was, $1 \mathrm{mmol}$ acetophenone and $1 \mathrm{~mL} \mathrm{HBr}$ were mixed and stirred in $1 \mathrm{~mL}$ DMSO at $55^{\circ} \mathrm{C}$ for $10-12 \mathrm{~h}$, then conducted with $1 \mathrm{~mL}$ aqueous ammonia at $0-5{ }^{\circ} \mathrm{C}$ and stirred for $1 \mathrm{~h}$.

Having the optimal reaction conditions established, we explored the scope of this cascade reaction. An array of aryl methyl ketones were examined. The results were shown in Table 2. Aromatic methyl ketones 1a-i bearing electron-neutral, electron-withdrawing or electrondonating substitutions at the benzene ring proceeded well to give good yields (Table 1, entries 1-9). The position of the substitution at benzene ring had little effect on the yield. The aryl methyl ketone with ortho group on the aromatic ring gave relatively lower yield (Table 1, entry 4), which indicated that steric hindrance influenced the reaction negatively. Polycyclic aromatic methyl ketones like $\mathbf{1 w}$ and $\mathbf{1 x}$ were also viable substrates and afforded the corresponding products in comparable yields. When a range of heteroaromatic methyl ketones were employed as the substrates, the corresponding products were obtained in moderate yields (Table 2, entries 10-16). Importantly, most products could precipitate from the solution with high purity, which made this process easy to be industrialized. It was a pity that no desired products were observed when 3-acetyl pyrrole (Table 2, entry 13) and 3-acetyl indole (Table 2 , entry 20) were employed as the substrates. It reflected that the DMSO oxidation of acetyl pyrrole was unworkable, which may have resulted from some side reactions such as nucleophilic attack at the pyrrole ring. ${ }^{29}$

Table 2. Reaction scope of aromatic ketones ${ }^{\mathrm{a}}$

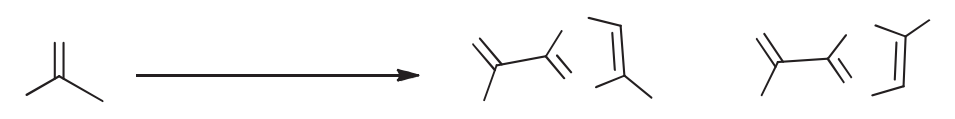

\begin{tabular}{cccc}
\hline Entry & $\mathrm{Ar}$ & Product & Yield $^{\mathrm{b}}(\%)$ \\
\hline 1 & $\mathrm{Ph}$ & $\mathbf{2 a}$ and $\mathbf{2 a}^{\prime}$ & 83 \\
2 & $3-\mathrm{FC}_{6} \mathrm{H}_{4}$ & $\mathbf{2 b}$ and $\mathbf{2} \mathbf{b}^{\prime}$ & 76 \\
3 & $3-\mathrm{ClC}_{6} \mathrm{H}_{4}$ & $\mathbf{2 c}$ and $\mathbf{2 \mathbf { c } ^ { \prime }}$ & 79 \\
4 & $2-\mathrm{BrC}_{6} \mathrm{H}_{4}$ & $\mathbf{2 d}$ and $\mathbf{2 d ^ { \prime }}$ & 45 \\
5 & $3-\mathrm{BrC}_{6} \mathrm{H}_{4}$ & $\mathbf{2 e}$ and $\mathbf{2 \mathbf { e } ^ { \prime }}$ & 86 \\
6 & $4-\mathrm{BrC}_{6} \mathrm{H}_{4}$ & $\mathbf{2 f}$ and $\mathbf{2 \mathbf { f } ^ { \prime }}$ & 68 \\
7 & $4-\mathrm{MeC}_{6} \mathrm{H}_{4}$ & $\mathbf{2 g}$ and $\mathbf{2 \mathbf { g } ^ { \prime }}$ & 81 \\
8 & $4-\mathrm{OHC}_{6} \mathrm{H}_{4}$ & $\mathbf{2 h}$ and $\mathbf{2 \mathbf { h } ^ { \prime }}$ & 51 \\
9 & $4-\mathrm{EtOC}_{4}$ & $\mathbf{2 i}$ and $\mathbf{2 i}$ & 85
\end{tabular}




\begin{tabular}{|c|c|c|c|}
\hline 10 & 2-Pyridyl & $2 \mathrm{j}$ and $2 \mathrm{j}^{\prime}$ & 59 \\
\hline 11 & 3-Pyridyl & $2 \mathbf{k}$ and $2 \mathbf{k}^{\prime}$ & 61 \\
\hline \multicolumn{4}{|c|}{ Table 2. Continued } \\
\hline Entry & $\mathrm{Ar}$ & Product & Yield $^{\mathrm{b}}(\%)$ \\
\hline 12 & 4-Pyridyl & $2 \mathrm{l}$ and $2 \mathrm{I}^{\prime}$ & 65 \\
\hline 13 & 3-Pyrryl & $2 \mathrm{~m}$ and $2 \mathrm{~m}^{\prime}$ & 0 \\
\hline 14 & 2-Furyl & 2n and $2 n^{\prime}$ & 51 \\
\hline 15 & 2-Thienyl & 20 and $20^{\prime}$ & 78 \\
\hline 16 & 2-Thiazolyl & $2 \mathbf{p}$ and $2 \mathbf{p}^{\prime}$ & 55 \\
\hline 17 & 1-Naphthyl & $\mathbf{2 q}$ and $\mathbf{2} \mathbf{q}^{\prime}$ & 11 \\
\hline 18 & 2-Naphthyl & $2 r$ and $2 r^{\prime}$ & 41 \\
\hline 19 & 3-Quinolyl & $2 s$ and $2 s^{\prime}$ & 66 \\
\hline 20 & 3-Indolyl & $\mathbf{2 t}$ and $\mathbf{2} \mathbf{t}^{\prime}$ & 0 \\
\hline 21 & 2-Benzofuryl & $\mathbf{2 u}$ and $\mathbf{2} \mathbf{u}^{\prime}$ & 53 \\
\hline 22 & 2-Benzothiophenyl & $2 \mathbf{v}$ and $2 \mathbf{v}^{\prime}$ & 56 \\
\hline 23 & 3-Benzothiophenyl & $2 \mathbf{w}$ and $2 \mathbf{w}^{\prime}$ & 81 \\
\hline 24 & 9-Phenanthryl & $2 \mathbf{x}$ and $2 \mathbf{x}^{\prime}$ & 71 \\
\hline
\end{tabular}

${ }^{a}$ Reaction was performed with acetophenone $(1 \mathrm{mmol})$ and $\mathrm{HBr}(1 \mathrm{~mL})$ in DMSO (1 $\mathrm{mL})$ at $55^{\circ} \mathrm{C}$ for $10-12 \mathrm{~h}$, then conducted with aqueous ammonia $(1 \mathrm{~mL})$ at $0-5{ }^{\circ} \mathrm{C}$ for $1 \mathrm{~h}$.

${ }^{\mathrm{b}}$ Isolated yields.

It was reported that the 2,4 and 2,5 isomers of AAIs could be differentiated by NMR, but there were no statements on their analysis using HPLC. ${ }^{28}$ We used different stationary phase such as ODS C18, pentafluorophenyl and $\beta$-cyclodextrin to analyze product $\mathbf{2 k}$ and $\mathbf{2} \mathbf{k}^{\prime}$. The results showed that compound $\mathbf{2} \mathbf{k}$ and $\mathbf{2} \mathbf{k}^{\prime}$ coincided with each other to form a single chromatographic peak with high purity, which indicated that the two isomers of AAIs could not be separated by HPLC column. Furthermore, the products of substrates $\mathbf{1} \mathbf{j}$ and $\mathbf{1 k}$ were confirmed by X-ray crystallography (Figure 2). ${ }^{31}$ The X-ray molecular structure revealed that the crystals of their products only had 2,4-isomers in solid form. This result confirmed the more stable configuration of AAI to be 2,4-isomer.

In the procedure of synthesizing AAIs, we determined the main by-products of this process to be 2-hydroxy-3,6-diaryl-pyrazines. They could be precipitated from the solvents 24 hours later after AAIs' filtering. However, owing to the low solubility, it was very difficult to characterize all of them by NMR. The identified 2-hydroxy-3,6-diaryl-pyrazines were shown in Table 3, others could be detected by HRMS as the isomers of their corresponding AAIs. 


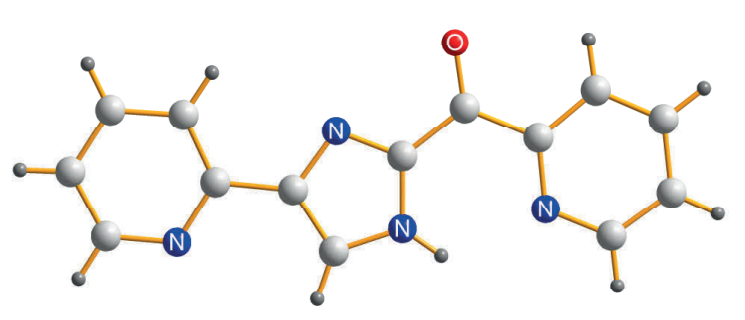

2j

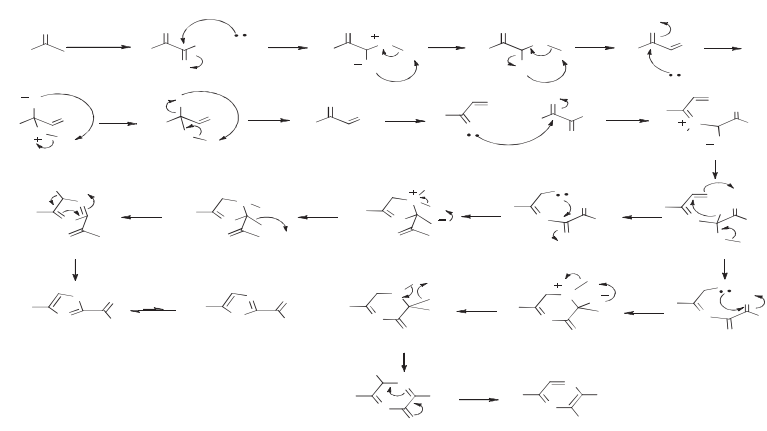

Figure 2. The X-ray molecular structure of $\mathbf{2 j}$ and $\mathbf{2 k}$.

Various conditions of the cascade procedure indicated that the yields of 2-hydroxy-3,6diaryl-pyrazines increased with increasing temperature. The results suggested that this procedure was thermodynamically controlled. Furthermore, the yields of pyrazines were higher when ammonium acetate was used in the Debus condensation. In summary, aqueous ammonia and low temperature were beneficial to produce imidazoles, while ammonium acetate and high temperature were beneficial to produce pyrazines in this cascade process. The structure of product 3p was also confirmed by X-ray crystallography. ${ }^{31}$ The bond length of the phenolic hydroxy was shorter than normal hydroxyl (1.41-1.44), being a medium between hydroxyl and carbonyl. Probably the hydroxy on $\mathbf{3 p}$ has a equilibrium between keto form and the enol form.

Table 3. Reaction scope of 2-hydroxy-3,6-diaryl-pyrazine ${ }^{\mathrm{a}}$

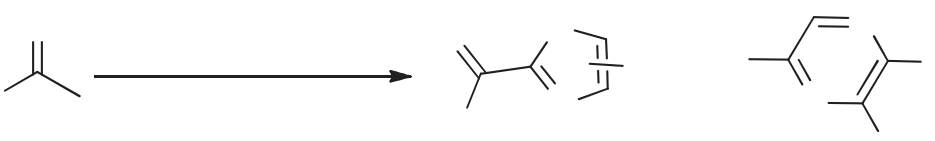

\begin{tabular}{cccc}
\hline Entry & $\mathrm{Ar}$ & Product/Yield $^{\mathrm{b}}(\%)$ & Product/Yield $^{\mathrm{b}}(\%)$ \\
\hline 1 & $\mathrm{Ph}$ & $\mathbf{2 a}, \mathbf{2} \mathbf{a}^{\prime} / 73$ & $\mathbf{3 a} / 7$ \\
2 & 3-ClC $\mathrm{H}_{4}$ & $\mathbf{2 c}, \mathbf{2} \mathbf{c}^{\prime} / 43$ & $\mathbf{3 c} / 36$ \\
3 & 2-Pyridyl & $\mathbf{2 j}, \mathbf{2} \mathbf{j}^{\prime} / 34$ & $\mathbf{3 j} / 15$ \\
4 & 3-Pyridyl & $\mathbf{2 k}, \mathbf{2} \mathbf{k}^{\prime} / 41$ & $\mathbf{3 k} / 19$ \\
5 & 4-Pyridyl & $\mathbf{2 l ,} \mathbf{2} \mathbf{l}^{\prime} / 37$ & $\mathbf{2 l} / 26$ \\
6 & 2-Thiazolyl & $\mathbf{2 p}, \mathbf{2} \mathbf{p}^{\prime} / 45$ & $\mathbf{3 p} / 8$ \\
\hline
\end{tabular}

${ }^{\text {a }}$ Reaction was performed with acetophenone $(1 \mathrm{mmol})$ and $\mathrm{HBr}(1 \mathrm{~mL})$ in DMSO $(1 \mathrm{~mL})$ at $55^{\circ} \mathrm{C}$ for $10-12 \mathrm{~h}$, then conducted with ammonium acetate $(100 \mathrm{mg})$ at $80^{\circ} \mathrm{C}$ for $1 \mathrm{~h}$.

${ }^{\mathrm{b}}$ Isolated yields. 


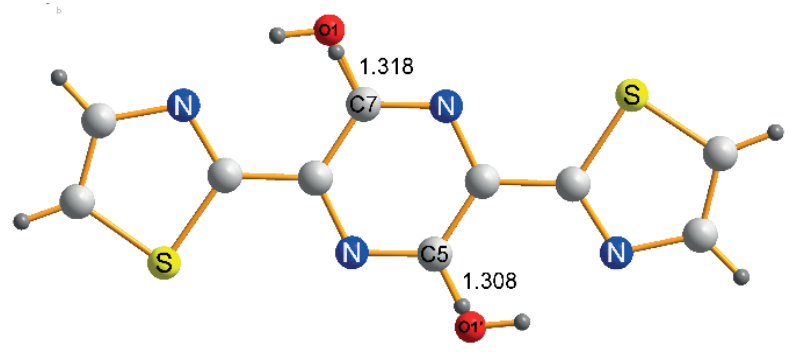

Figure 3. The X-ray molecular structure of 3p.

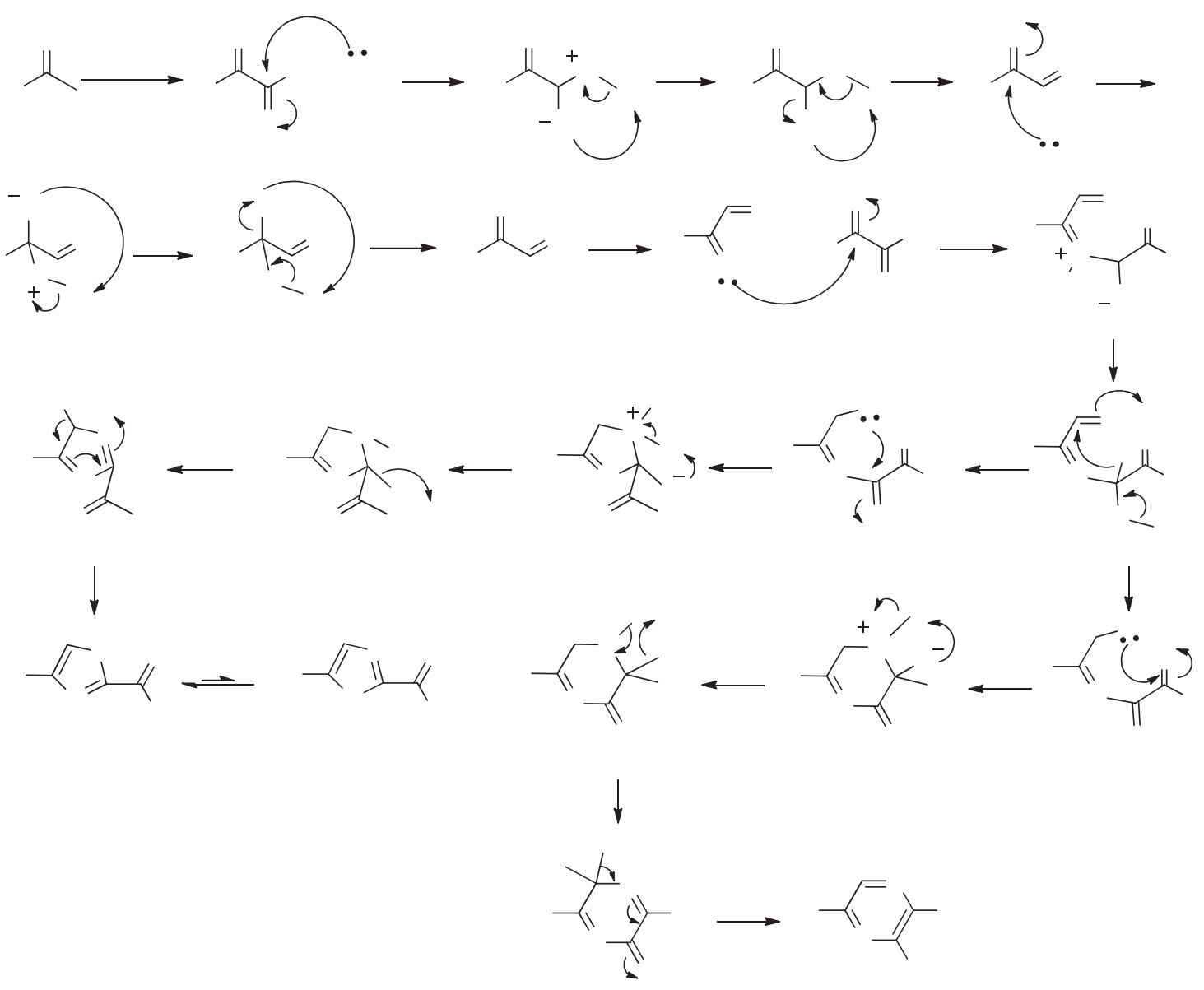

Scheme 2. A plausible mechanism for imidazole and pyrazine synthesis.

Referring to the previous literature,$^{30}$ the possible mechanism for this procedure was illustrated with the example of acetophenone and aqueous ammonia (as shown in Scheme 2). As to intermediate 13a, the electron doublet of $\mathrm{N}$ atom could attack carbonyl group $\mathbf{1}$ to form imidazole $\mathbf{1 b}$ and $\mathbf{1} \mathbf{b}$ ' gradually (Scheme 2, route 1), and also could form pyrazine $\mathbf{1 c}$ by attacking carbonyl group 2 (Scheme 2, route 2). As the electropositivity of carbonyl group 1 
was higher than carbonyl group $\mathbf{2}$, the main product was imidazole $\mathbf{1 b}$ and $\mathbf{1} \mathbf{b}$ '. However, with the rise of temperature, the probability of attacking carbonyl group 2 increased. That's why the yields of 2-hydroxy-3,6-diaryl-pyrazines increased with temperature.

\section{Conclusions}

In summary, we report a cascade procedure for the synthesis of (4 or 5)-aryl-2-aryloyl-1Himidazoles and 2-hydroxy-3,6-diaryl-pyrazines from aryl methyl ketones. The mechanism was also conjectured. Owing to the simple starting materials, mild conditions, easy operation, high bioactivity of imidazole and pyrazine derivatives, this protocol not only meets the demand of commercial application, but also has great potential in medicinal chemistry.

\section{Experimental Section}

General. All reagents and solvents were purchased from J\&K Chemical Co. and used without further purification. Melting points were determined on a SGW X-4 micro melting point instrument. ${ }^{1} \mathrm{H}$ and ${ }^{13} \mathrm{C}$ NMR spectra were recorded on Bruker 400 or $500 \mathrm{MHz}$ spectrometer. HPLC impurities were determined on a Shimadzu 10A chromatographic instrument. IR spectra were obtained on a Perkin Elmer FT-IR system. UV spectra were obtained on a TU1810 ultraviolet visible spectrophotometer. HRMS spectra were obtained by a LTQ Orbitrap Discovery spectrometer from Thermo.

\section{General procedure for the synthesis of compounds 2}

$1 \mathrm{mmol}$ aryl methyl ketone and $1 \mathrm{~mL}$ aqueous $\mathrm{HBr}(48 \%)$ were mixed in $1 \mathrm{~mL}$ DMSO. The mixture was stirred at $55{ }^{\circ} \mathrm{C}$ for 10-12 h. After cooling in ice bath, aqueous ammonia $(1 \mathrm{~mL}$, $28 \%$ ) was added to the solution. The mixture was stirred at $0-5{ }^{\circ} \mathrm{C}$ for $1 \mathrm{~h}$. The obtained solid was filtered off to give AAI.

\section{General procedure for the synthesis of compounds 3}

$1 \mathrm{mmol}$ aryl methyl ketone and $1 \mathrm{~mL}$ aqueous $\mathrm{HBr}(48 \%)$ were mixed in $1 \mathrm{~mL}$ DMSO. The mixture was stirred at $55^{\circ} \mathrm{C}$ for $10-12 \mathrm{~h}$. After heating-up to $80{ }^{\circ} \mathrm{C}$, ammonium acetate $(100$ $\mathrm{mg}$ ) was added to the solution. The mixture was stirred for $1 \mathrm{~h}$. The obtained solid was filtered off. 2-Hydroxy-3,6-diarylpyrazine could be filtered from the filter liquor 24 hours later.

Phenyl-(4-phenyl-1H-imidazol-2-yl)methanone (2a) and phenyl-(5-phenyl- $1 H$-imidazol2-yl)methanone (2a'). Yellow solid. Mp: 229-230 ${ }^{\circ} \mathrm{C}$. NMR (400 MHz, DMSO- $\left.d_{6}\right): \delta 13.80$ (s, 1H), $13.63(\mathrm{~s}, 1 \mathrm{H}), 8.60(\mathrm{~d}, J 6.8 \mathrm{~Hz}, 2 \mathrm{H}), 8.48$ (s, 1H), 8.09 (s, 1H), 7.95 (d, J $7.6 \mathrm{~Hz}$, 
2.7H), $7.70(\mathrm{t}, J$ 7.2 Hz, 1.3H), $7.61(\mathrm{t}, J 7.6 \mathrm{~Hz}, 2.5 \mathrm{H}), 7.44(\mathrm{t}, J 7.2 \mathrm{~Hz}, 2.7 \mathrm{H}), 7.31(\mathrm{~d}, J$ $6.8 \mathrm{~Hz}, 1 \mathrm{H}) .{ }^{13} \mathrm{C}$ NMR $\left(100 \mathrm{MHz}, \mathrm{DMSO}-d_{6}\right): \delta 180.8,144.7,142.9,136.0,133.7,133.1$, 130.7, 128.7, 128.3, 127.2, 124.9, 118.7. IR (KBr, cm $\left.{ }^{-1}\right): 3271,1620,1571,1454,1439,1292$, 1273, 1169, 904, 869, 764, 732, 690, 646. UV/Vis (acetonitrile): $\lambda_{\max }(\varepsilon)=197$ (1.846), 257 (1.090), 337 (0.798) nm. HPLC purity: 98.08\%. HRMS (ESI-Orbitrap) $m / z:[\mathrm{M}+\mathrm{H}]^{+}$Calcd for $\mathrm{C}_{16} \mathrm{H}_{12} \mathrm{~N}_{2} \mathrm{O}+\mathrm{H}^{+} 249.10224$; Found 249.10225.

(3-Fluorophenyl)-[4-(3-fluorophenyl)-1H-imidazol-2-yl]methanone $\quad(2 \mathrm{~b}) \quad$ and (3fluorophenyl)[5-(3-fluorophenyl)-1H-imidazol-2-yl]methanone (2b'). Yellow solid. Mp: 195-197 ${ }^{\circ} \mathrm{C}$. NMR (400 MHz, DMSO-d $\left.)_{6}\right): \delta 13.80(\mathrm{~s}, 1 \mathrm{H}), 8.44$ (d, $\left.J 6.0 \mathrm{~Hz}, 1 \mathrm{H}\right), 8.37$ (d, $J$ $10.0 \mathrm{~Hz}, 1 \mathrm{H}), 8.20(\mathrm{~s}, 1 \mathrm{H}), 7.80(\mathrm{~d}, J 7.6 \mathrm{~Hz}, 1 \mathrm{H}), 7.76(\mathrm{~d}, J 9.2 \mathrm{~Hz}, 1 \mathrm{H}), 7.68$ (dd, J $14.0 \mathrm{~Hz}$, $J 8.0 \mathrm{~Hz}, 1 \mathrm{H}), 7.57(\mathrm{td}, J 8.4 \mathrm{~Hz}, J 2.0 \mathrm{~Hz}, 1 \mathrm{H}), 7.49(\mathrm{dd}, J 14.4 \mathrm{~Hz}, J 8.0 \mathrm{~Hz}, 1 \mathrm{H}), 7.14(\mathrm{t}, J$ $7.2 \mathrm{~Hz}, 1 \mathrm{H}) .{ }^{13} \mathrm{C}$ NMR $\left(100 \mathrm{MHz}, \mathrm{DMSO}-d_{6}\right): \delta 179.22,179.20,163.9,162.9,161.5,160.5$, $144.5,141.8,137.9,137.8,136.0,130.8,130.7,130.6,130.5,126.9,126.8,120.9,120.2$, 120.0, 117.2, 117.0, 114.0, 113.8, 111.5, 111.3. IR $\left(\mathrm{KBr}, \mathrm{cm}^{-1}\right): 3424,3287,1617,1581$, $1468,1446,1289,1263,1237,1173,855,820,789,766,681$. UV/Vis (acetonitrile): $\lambda_{\max }(\varepsilon)$ $=208$ (1.723), 255 (2.093), 337 (1.503) nm. HPLC purity: 96.37\%. HRMS (ESI-Orbitrap) $m / z:[\mathrm{M}+\mathrm{H}]^{+}$Calcd for $\mathrm{C}_{16} \mathrm{H}_{10} \mathrm{~F}_{2} \mathrm{~N}_{2} \mathrm{O}+\mathrm{H}^{+}$285.08340; Found 285.08325.

(3-Chlorophenyl)-[4-(3-chlorophenyl)-1H-imidazol-2-yl]methanone (2c) and (3chlorophenyl)-[5-(3-chlorophenyl)-1H-imidazol-2-yl]methanone (2c'). Yellow solid. Mp: 229-233 ${ }^{\circ} \mathrm{C}$. NMR (400 MHz, DMSO- $\left.d_{6}\right): \delta 13.96$ (s, $\left.0.2 \mathrm{H}\right), 13.79$ (s, 1H), $8.56(\mathrm{~s}, 0.7 \mathrm{H})$, $8.52(\mathrm{~d}, J 7.6 \mathrm{~Hz}, 1 \mathrm{H}), 8.37$ (d, J $8.0 \mathrm{~Hz}, 0.2 \mathrm{H}), 8.22$ (d, J 2.0 Hz, 1H), 8.12 (s, 0.2H), 7.95 $(\mathrm{s}, 1 \mathrm{H}), 7.89(\mathrm{~m}, 1.3 \mathrm{H}), 7.75(\mathrm{~m}, 1 \mathrm{H}), 7.64(\mathrm{t}, J 8.0 \mathrm{~Hz}, 1 \mathrm{H}), 7.59(\mathrm{~d}, J 8.0 \mathrm{~Hz}, 0.1 \mathrm{H}), 7.45(\mathrm{~m}$, $1.4 \mathrm{H}), 7.33(\mathrm{~d}, J 7.6 \mathrm{~Hz}, 1 \mathrm{H}) .{ }^{13} \mathrm{C}$ NMR (100 MHz, DMSO- $\left.d_{6}\right): \delta 179.4,179.2,145.7,144.5$, $141.6,137.8,137.6,135.7,134.6,133.9,133.7,133.1,133.0,132.9,132.7,130.8,130.7$, $130.6,130.5,130.3,130.2,130.1,129.3,129.1,128.1,127.0,125.4,124.4,124.3,123.4$, 120.1. IR (KBr, cm $\left.{ }^{-1}\right): 3446,3286,1619,1563,1455,1434,1309,1280,1270,1164,1099$, 1080, 792, 764, 735, 684. UV/Vis (acetonitrile): $\lambda_{\max }(\varepsilon)=219$ (2.348), 254 (2.544), 337 (2.023) nm. HPLC purity: 98.42\%. HRMS (ESI-Orbitrap) $m / z:[\mathrm{M}+\mathrm{H}]^{+}$Calcd for $\mathrm{C}_{16} \mathrm{H}_{10} \mathrm{Cl}_{2} \mathrm{~N}_{2} \mathrm{O}+\mathrm{H}^{+}$317.02429; Found 317.02429.

(2-Bromophenyl)-[4-(2-bromophenyl)-1H-imidazol-2-yl]methanone (2d) and (2bromophenyl)-[5-(2-bromophenyl)-1H-imidazol-2-yl)methanone (2d'). Yellow solid. Mp: 184-186 ${ }^{\circ} \mathrm{C}$. NMR (400 MHz, DMSO-d $): \delta 13.96(\mathrm{~s}, 1 \mathrm{H}), 8.06$ (d, $\left.J 1.6 \mathrm{~Hz}, 1 \mathrm{H}\right), 7.75$ (m, 2.8H), $7.70(\mathrm{~d}, J 5.2 \mathrm{~Hz}, 1.1 \mathrm{H}), 7.66(\mathrm{~m}, 0.9 \mathrm{H}), 7.51(\mathrm{~m}, 3 \mathrm{H}), 7.41(\mathrm{t}, J 7.6 \mathrm{~Hz}, 1.3 \mathrm{H}), 7.24(\mathrm{t}$, $J$ 7.6 Hz, 1.1H). ${ }^{13} \mathrm{C}$ NMR (100 MHz, DMSO- $\left.d_{6}\right): \delta 184.0,143.6,141.4,139.1,133.8,133.4$, 132.9, 132.0, 130.8, 130.5, 129.2, 127.8, 127.3, 122.2, 120.6, 119.4. IR (KBr, $\left.\mathrm{cm}^{-1}\right): 3466$, $3270,1648,1450,1395,1296,1136,1017,911,744,725,624$. UV/Vis (acetonitrile): $\lambda_{\max }(\varepsilon)$ $=221$ (2.339), 318 (2.166) nm. HPLC purity: 99.26\%. HRMS (ESI-Orbitrap) $m / z:[\mathrm{M}+\mathrm{H}]^{+}$ Calcd for $\mathrm{C}_{16} \mathrm{H}_{10} \mathrm{Br}_{2} \mathrm{~N}_{2} \mathrm{O}+\mathrm{H}^{+}$404.92326; Found 404.92328. 
(3-Bromophenyl)-[4-(3-bromophenyl)-1H-imidazol-2-yl]methanone $\quad(2 e) \quad$ and $\quad$ (3bromophenyl)-[(5-(3-bromophenyl)-1H-imidazol-2-yl]methanone (2e'). Orange solid. Mp: 148-152 ${ }^{\circ} \mathrm{C}$. NMR (400 MHz, DMSO-d $\left.)_{6}\right): \delta 13.75(\mathrm{~s}, 1 \mathrm{H}), 8.71(\mathrm{~s}, 0.8 \mathrm{H}), 8.54(\mathrm{~d}, J 7.6 \mathrm{~Hz}$, $1 \mathrm{H}), 8.19(\mathrm{~s}, 1 \mathrm{H}), 8.14(\mathrm{~s}, 1.1 \mathrm{H}), 7.94(\mathrm{~d}, J 7.6 \mathrm{~Hz}, 1.1 \mathrm{H}), 7.91(\mathrm{dd}, J 8.0 \mathrm{~Hz}, J 1.2 \mathrm{~Hz}, 1 \mathrm{H})$, $7.59(\mathrm{t}, J 8.0 \mathrm{~Hz}, 1.1 \mathrm{H}), 7.50(\mathrm{~d}, J 8.0 \mathrm{~Hz}, 1 \mathrm{H}), 7.41(\mathrm{t}, J 8.0 \mathrm{~Hz}, 1.1 \mathrm{H}) .{ }^{13} \mathrm{C} \mathrm{NMR}(100 \mathrm{MHz}$, DMSO- $\left.d_{6}\right): \delta 179.1,144.6,137.8,135.7,133.1,130.9,130.6,130.0,129.6,127.4,123.8$, 122.3, 121.5. IR ( $\left.\mathrm{KBr}, \mathrm{cm}^{-1}\right): 3447,3285,1618,1557,1452,1429,1278,1269,1164,1070$, 920, 790, 738, 680. UV/Vis (acetonitrile): $\lambda_{\max }(\varepsilon)=225$ (2.463), 258 (2.540), 336 (1.990) nm. HPLC purity: $98.18 \%$. HRMS (ESI-Orbitrap) $m / z:[\mathrm{M}+\mathrm{H}]^{+}$Calcd for $\mathrm{C}_{16} \mathrm{H}_{10} \mathrm{Br}_{2} \mathrm{~N}_{2} \mathrm{O}+\mathrm{H}^{+}$ 404.92326; Found 404.92316.

(4-Bromophenyl)-[4-(4-bromophenyl)-1H-imidazol-2-yl]methanone (2f) and (4bromophenyl)-[5-(4-bromophenyl)-1H-imidazol-2-yl]methanone (2f'). Yellow solid. Mp: 257-260 ${ }^{\circ} \mathrm{C}$. NMR (400 MHz, DMSO-d $): \delta 13.90(\mathrm{~s}, 0.2 \mathrm{H}), 13.74(\mathrm{~s}, 0.9 \mathrm{H}), 8.52(\mathrm{~d}, J 8.0$ $\mathrm{Hz}, 1.9 \mathrm{H}), 8.44$ (s, 0.3H), $8.16(\mathrm{~s}, 1 \mathrm{H}), 7.90$ (d, J 8.0 Hz, 2.4H), 7.83 (d, J 8.4 Hz, 2.2H), 7.62 $(\mathrm{d}, J 8.0 \mathrm{~Hz}, 2.2 \mathrm{H}) .{ }^{13} \mathrm{C} \mathrm{NMR}\left(100 \mathrm{MHz}, \mathrm{DMSO}-d_{6}\right): \delta 179.7,144.6,141.9,134.9,132.8$, 132.6, 131.6, 131.5, 127.5, 126.9, 120.2, 119.5. IR (KBr, cm$\left.{ }^{-1}\right): 3418,3270,1616,1579$, 1451, 1291, 1167, 1071, 1012, 904, 832, 767, 644. UV/Vis (acetonitrile): $\lambda_{\max }(\varepsilon)=265$ (0.866), 342 (0.518) nm. HPLC purity: 97.62\%. HRMS (ESI-Orbitrap) $m / z:[\mathrm{M}+\mathrm{H}]^{+}$Calcd for $\mathrm{C}_{16} \mathrm{H}_{10} \mathrm{Br}_{2} \mathrm{~N}_{2} \mathrm{O}+\mathrm{H}^{+} 404.92326$; Found 404.92279.

p-Tolyl-[4-(p-tolyl)-1H-imidazol-2-yl]methanone $\quad(2 \mathrm{~g}) \quad$ and $\quad p$-tolyl-[5-(p-tolyl)-1Himidazol-2-yl]methanone $\left(\mathbf{2 g}^{\prime}\right)$. Yellow solid. Mp: 207-211 ${ }^{\circ} \mathrm{C}$. NMR (400 MHz, DMSO$\left.d_{6}\right): \delta 13.66(\mathrm{~s}, 0.3 \mathrm{H}), 13.52(\mathrm{~s}, 1 \mathrm{H}), 8.52(\mathrm{~d}, J 8.4 \mathrm{~Hz}, 2 \mathrm{H}), 8.40(\mathrm{~d}, J 8.0 \mathrm{~Hz}, 0.6 \mathrm{H}), 7.99(\mathrm{~s}$, $1 \mathrm{H}), 7.84(\mathrm{~d}, J 8.4 \mathrm{~Hz}, 0.6 \mathrm{H}), 7.81(\mathrm{~d}, J 8.0 \mathrm{~Hz}, 2.1 \mathrm{H}), 7.71(\mathrm{~s}, 0.3 \mathrm{H}), 7.40(\mathrm{~d}, J 8.0 \mathrm{~Hz}$, 2.1H), $7.36(\mathrm{~d}, J 8.0 \mathrm{~Hz}, 0.7 \mathrm{H}), 7.26(\mathrm{~d}, J 8.4 \mathrm{~Hz}, 0.7 \mathrm{H}), 7.23(\mathrm{~d}, J 7.6 \mathrm{~Hz}, 2 \mathrm{H}), 2.41(\mathrm{~m}$, 4.1H), $2.32(\mathrm{~s}, 4 \mathrm{H}) .{ }^{13} \mathrm{C}$ NMR (100 MHz, DMSO-d $): \delta 180.4,180.3,145.7,144.7,143.5$, $143.3,142.9,137.8,136.3,135.7,133.6,133.5,131.0,130.8,130.7,129.5,129.2,129.0$, $128.8,128.4,125.9,125.7,124.8,118.0,21.3,20.9 . \mathrm{IR}\left(\mathrm{KBr}, \mathrm{cm}^{-1}\right): 3419,3273,1616,1602$, 1450, 1287, 1269, 1168, 904, 822, 762. UV/Vis (acetonitrile): $\lambda_{\max }(\varepsilon)=205$ (1.379), 260 (1.413), 343 (1.078) nm. HPLC purity: 98.96\%. HRMS (ESI-Orbitrap) $m / z:[\mathrm{M}+\mathrm{H}]^{+}$Calcd for $\mathrm{C}_{18} \mathrm{H}_{16} \mathrm{~N}_{2} \mathrm{O}+\mathrm{H}^{+} 277.13354$; Found 277.13354.

(4-Hydroxyphenyl)-[4-(4-hydroxyphenyl)-1H-imidazol-2-yl]methanone (2h) and (4hydroxyphenyl)-[(5-(4-hydroxyphenyl)-1H-imidazol-2-yl]methanone (2h'). Yellow solid. Mp: 317-322 ${ }^{\circ} \mathrm{C}$. NMR (400 MHz, DMSO-d $): \delta 13.37(\mathrm{~s}, 0.4 \mathrm{H}), 13.29(\mathrm{~s}, 1 \mathrm{H}), 10.42$ (s, $1.3 \mathrm{H}), 9.73(\mathrm{~s}, 0.4 \mathrm{H}), 9.46(\mathrm{~s}, 1 \mathrm{H}), 8.60(\mathrm{~d}, J 8.4 \mathrm{~Hz}, 1.9 \mathrm{H}), 8.46$ (d, J $8.8 \mathrm{~Hz}, 0.8 \mathrm{H}), 7.78$ (s, $1 \mathrm{H}), 7.76(\mathrm{~s}, 0.4 \mathrm{H}), 7.73(\mathrm{~s}, 1.4 \mathrm{H}), 7.71(\mathrm{~s}, 1.1 \mathrm{H}), 7.55(\mathrm{~s}, 0.5 \mathrm{H}), 6.94(\mathrm{~s}, 1 \mathrm{H}), 6.91(\mathrm{~m}, 1.4 \mathrm{H})$, $6.88(\mathrm{~s}, 0.4 \mathrm{H}), 6.83(\mathrm{~m}, 1.8 \mathrm{H}), 6.80(\mathrm{~s}, 1 \mathrm{H}) .{ }^{13} \mathrm{C}$ NMR $\left(100 \mathrm{MHz}, \mathrm{DMSO}-d_{6}\right): \delta 178.9,178.8$, 162.2 , 161.9, 157.6, 156.6, 145.4, 144.7, 142.9, 135.6, 133.4, 133.2, 127.5, 127.2, 127.2, $127.0,126.2,124.9,119.7,116.2,115.6,115.4,115.0,114.9 . \mathrm{IR}\left(\mathrm{KBr}, \mathrm{cm}^{-1}\right): 3383,3253$, $2588,1616,1600,1574,1438,1418,1274,1198,1180,1162,910,826,776,758$. UV/Vis 
(acetonitrile): $\lambda_{\max }(\varepsilon)=260$ (0.567), 298 (0.471), 351 (0.576) nm. HPLC purity: 99.14\%. HRMS (ESI-Orbitrap) m/z: $[\mathrm{M}+\mathrm{H}]^{+}$Calcd for $\mathrm{C}_{16} \mathrm{H}_{12} \mathrm{~N}_{2} \mathrm{O}_{3}+\mathrm{H}^{+}$281.09207; Found 281.09164.

(4-Ethoxyphenyl)-[4-(4-ethoxyphenyl)-1H-imidazol-2-yl]methanone (2i) and (4ethoxyphenyl)-[5-(4-ethoxyphenyl)-1H-imidazol-2-yl]methanone (2i'). Orange solid. Mp: 192-194 ${ }^{\circ} \mathrm{C}$. NMR (400 MHz, DMSO- $\left.d_{6}\right): \delta 13.53$ (s, 0.4H), 13.41 (s, 1H), 8.69 (d, J 8.8 Hz, 2H), $8.55(\mathrm{~d}, J 8.8 \mathrm{~Hz}, 0.7 \mathrm{H}), 7.90(\mathrm{~s}, 1.1 \mathrm{H}), 7.89(\mathrm{~s}, 0.4 \mathrm{H}), 7.87$ (s, 0.4H), 7.84 (d, J $8.8 \mathrm{~Hz}$, 2.1H), $7.64(\mathrm{~s}, 0.4 \mathrm{H}), 7.12(\mathrm{~d}, J 8.4 \mathrm{~Hz}, 2.2 \mathrm{H}), 7.08(\mathrm{~d}, J 8.8 \mathrm{~Hz}, 0.8 \mathrm{H}), 7.01$ (s, 0.4H), 6.98 $(\mathrm{d}, J \quad 8.4 \mathrm{~Hz}, 2.5 \mathrm{H}), 4.16(\mathrm{~m}, 3.1 \mathrm{H}), 4.06(\mathrm{~m}, 3.1 \mathrm{H}), 1.36(\mathrm{~m}, 9.2 \mathrm{H}) .{ }^{13} \mathrm{C} \mathrm{NMR}(100 \mathrm{MHz}$, DMSO- $\left.d_{6}\right): \delta 179.1,178.9,162.6,162.4,158.6,157.9,145.6,144.8,142.7,135.4,133.2$, 133.0, 128.7, 128.5, 127.6, 127.2, 126.4, 126.2, 121.2, 117.0, 114.8, 114.5, 114.1, 114.0, 63.6, 63.2, 63.0, 14.7, 14.7, 14.6. IR (KBr, $\left.\mathrm{cm}^{-1}\right): 3417,3263,1611,1557,1446,1284,1253,1159$, 1116, 1038, 903, 835, 672, 645. UV/Vis (acetonitrile): $\lambda_{\max }(\varepsilon)=262(0.794), 299(0.658)$, 351 (0.756) nm. HPLC purity: 95.84\%. HRMS (ESI-Orbitrap) $m / z:[\mathrm{M}+\mathrm{H}]^{+}$Calcd for $\mathrm{C}_{20} \mathrm{H}_{20} \mathrm{~N}_{2} \mathrm{O}_{3}+\mathrm{H}^{+} 337.15467$; Found 337.15466.

Pyridin-2-yl-[4-(pyridin-2-yl)-1H-imidazol-2-yl]methanone $(2 \mathrm{j})$ and pyridin-2-yl-[5(pyridin-2-yl)-1H-imidazol-2-yl]methanone (2j'). Yellow solid. Mp: 236-237 ${ }^{\circ} \mathrm{C}$. NMR (500 MHz, DMSO-d $): \delta 13.78$ (s, 0.9H), 8.82 (ddd, J $5.0 \mathrm{~Hz}, J 2.0 \mathrm{~Hz}, J 1.0 \mathrm{~Hz}, 1 \mathrm{H}), 8.58$ $(\mathrm{d}, J 3.5 \mathrm{~Hz}, 1 \mathrm{H}), 8.36(\mathrm{~d}, J 7.5 \mathrm{~Hz}, 1 \mathrm{H}), 8.10(\mathrm{td}, J 7.5 \mathrm{~Hz}, J 1.5 \mathrm{~Hz}, 1 \mathrm{H}), 8.02$ (s, $0.9 \mathrm{H})$, $8.00(\mathrm{~d}, J 7.5 \mathrm{~Hz}, 1 \mathrm{H}), 7.86(\mathrm{td}, J 7.5 \mathrm{~Hz}, J 1.0 \mathrm{~Hz}, 1 \mathrm{H}), 7.71(\mathrm{ddd}, J 7.5 \mathrm{~Hz}, J 5.0 \mathrm{~Hz}, J 1.0$ $\mathrm{Hz}, 1 \mathrm{H}), 7.31(\mathrm{t}, J 5.5 \mathrm{~Hz}, 1 \mathrm{H}) .{ }^{13} \mathrm{C}$ NMR (125 MHz, DMSO-d $): \delta 179.4,153.3,152.3$, $149.3,144.1,143.7,137.4,137.0,127.0,125.3,122.4,120.5,119.1 . \mathrm{IR}\left(\mathrm{KBr}, \mathrm{cm}^{-1}\right): 3403$, 3060, 1652, 1622, 1599, 1487, 1455, 1389, 1295, 1089, 996, 909, 781, 756, 618. UV/Vis (acetonitrile): $\lambda_{\max }(\varepsilon)=201$ (1.951), 248 (2.007), 342 (1.936) nm. HPLC purity: 98.47\%. HRMS (ESI-Orbitrap) $m / z$ : $[\mathrm{M}+\mathrm{H}]^{+}$Calcd for $\mathrm{C}_{14} \mathrm{H}_{10} \mathrm{~N}_{4} \mathrm{O}+\mathrm{H}^{+} 251.09274$; Found 251.09271 . Pyridin-3-yl-[4-(pyridin-3-yl)-1H-imidazol-2-yl]methanone (2k) and pyridin-3-yl-[5(pyridin-3-yl)-1H-imidazol-2-yl]methanone (2k'). Yellow solid. Mp: 237-240 ${ }^{\circ} \mathrm{C}$. NMR $\left(500 \mathrm{MHz}, \mathrm{DMSO}-d_{6}\right): \delta 14.13(\mathrm{~s}, 0.2 \mathrm{H}), 13.90(\mathrm{~s}, 1 \mathrm{H}), 9.65$ (d, J $\left.1.5 \mathrm{~Hz}, 0.9 \mathrm{H}\right), 9.54$ (s, $0.1 \mathrm{H}), 9.19(\mathrm{~s}, 0.1 \mathrm{H}), 9.17(\mathrm{~d}, J 1.5 \mathrm{~Hz}, 1 \mathrm{H}), 8.87(\mathrm{~m}, 2.2 \mathrm{H}), 8.73(\mathrm{~d}, J 8.0 \mathrm{~Hz}, 0.2 \mathrm{H}), 8.58$ (d, J 4.0 Hz, 0.2H), 8.52 (dd, J 4.5 Hz, J 1.5 Hz, 1H), 8.36 (d, J 8.0 Hz, 0.2H), 8.29 (m, 2H), $7.96(\mathrm{~s}, 0.1 \mathrm{H}), 7.66(\mathrm{dd}, J 7.5 \mathrm{~Hz}, J 5.0 \mathrm{~Hz}, 1 \mathrm{H}), 7.62(\mathrm{~m}, 0.2 \mathrm{H}), 7.51(\mathrm{~m}, 0.12 \mathrm{H}), 7.47$ (dd, $J$ $7.5 \mathrm{~Hz}, J 4.5 \mathrm{~Hz}, 1 \mathrm{H}) .{ }^{13} \mathrm{C}$ NMR $\left(125 \mathrm{MHz}, \mathrm{DMSO}-d_{6}\right): \delta 179.8,153.1,153.0,151.3,151.1$, $149.1,148.2,146.9,146.3,144.7,140.2,138.1,137.9,132.9,132.0,131.8,131.5,130.0$, 129.2, 124.7, 123.8, 123.5, 123.4, 120.0. IR (KBr, cm $\left.{ }^{-1}\right): 2360,2342,1622,1585,1420,1398$, 1308, 1270, 1154, 1026, 909, 811, 700. UV/Vis (acetonitrile): $\lambda_{\max }(\varepsilon)=203(0.461), 253$ (0.392), 335 (0.322) nm. HPLC purity: 97.02\%. HRMS (ESI-Orbitrap) $m / z:[\mathrm{M}+\mathrm{H}]^{+}$Calcd for $\mathrm{C}_{14} \mathrm{H}_{10} \mathrm{~N}_{4} \mathrm{O}+\mathrm{H}^{+} 251.09274$; Found 251.09273 .

Pyridin-4-yl-[4-(pyridin-4-yl)-1H-imidazol-2-yl]methanone (2l) and pyridin-4-yl-[5(pyridin-4-yl)-1H-imidazol-2-yl]methanone (2l'). Yellow solid. Mp: 346-347 ${ }^{\circ} \mathrm{C}$. NMR 
(400 MHz, DMSO-d $): \delta 14.26(\mathrm{~s}, 0.1 \mathrm{H}), 14.01(\mathrm{~s}, 1 \mathrm{H}), 8.87(\mathrm{~d}, J 5.6 \mathrm{~Hz}, 2.1 \mathrm{H}), 8.60(\mathrm{~d}, J$ $5.2 \mathrm{~Hz}, 2.2 \mathrm{H}), 8.40(\mathrm{~s}, 1 \mathrm{H}), 8.34(\mathrm{~d}, J 5.6 \mathrm{~Hz}, 1.9 \mathrm{H}), 8.22(\mathrm{~s}, 0.1 \mathrm{H}), 7.95(\mathrm{~s}, 0.1 \mathrm{H}), 7.88(\mathrm{~d}, J$ $5.6 \mathrm{~Hz}, 2 \mathrm{H}) .{ }^{13} \mathrm{C}$ NMR (100 MHz, DMSO- $\left.d_{6}\right): \delta 180.2,150.3,150.1,144.6,142.0,140.7$, 140.5, 123.6, 122.0, 119.3. IR (KBr, $\left.\mathrm{cm}^{-1}\right): 3422,2566,1896,1643,1608,1421,1407,1305$, $1273,1216,1014,911,822,761,682,636$. UV/Vis (acetonitrile): $\lambda_{\max }(\varepsilon)=195(1.376), 261$ (0.656), 330 (0.694) nm. HPLC purity: 99.43\%. HRMS (ESI-Orbitrap) $m / z:[\mathrm{M}+\mathrm{H}]^{+} \mathrm{Calcd}$ for $\mathrm{C}_{14} \mathrm{H}_{10} \mathrm{~N}_{4} \mathrm{O}+\mathrm{H}^{+} 251.09274$; Found 251.09279.

Furan-2-yl-[4-(furan-2-yl)-1H-imidazol-2-yl]methanone (2n) and furan-2-yl-[5-(furan-2yl)-1H-imidazol-2-yl]methanone (2n'). Black solid. Mp: 216-218 ${ }^{\circ} \mathrm{C}$. NMR $(500 \mathrm{MHz}$, DMSO-d $\left.)_{6}\right): \delta 13.95(\mathrm{~s}, 0.4 \mathrm{H}), 13.71(\mathrm{~s}, 1 \mathrm{H}), 8.30(\mathrm{~d}, J 3.5 \mathrm{~Hz}, 1 \mathrm{H}), 8.18(\mathrm{~d}, J 3.0 \mathrm{~Hz}, 0.4 \mathrm{H})$, $8.15(\mathrm{~s}, 1 \mathrm{H}), 8.14(\mathrm{~s}, 0.3 \mathrm{H}), 7.80(\mathrm{~s}, 0.4 \mathrm{H}), 7.77(\mathrm{~d}, J 2.5 \mathrm{~Hz}, 1 \mathrm{H}), 7.70(\mathrm{~s}, 0.9 \mathrm{H}), 7.54$ (s, $0.4 \mathrm{H}), 7.10(\mathrm{~d}, J 2.5 \mathrm{~Hz}, 0.4 \mathrm{H}), 6.83(\mathrm{dd}, J 3.5 \mathrm{~Hz}, J 1.5 \mathrm{~Hz}, 1 \mathrm{H}), 6.80$ (s, 0.4H), 6.79 (d, J $3.0 \mathrm{~Hz}, 1 \mathrm{H}), 6.64(\mathrm{~s}, 0.4 \mathrm{H}), 6.59$ (dd, J $3.0 \mathrm{~Hz}, J 1.5 \mathrm{~Hz}, 1 \mathrm{H}) .{ }^{13} \mathrm{C}$ NMR (100 MHz, DMSO$\left.d_{6}\right): \delta 168.2,150.0,148.8,143.8,142.1,135.4,123.4,118.0,112.9,111.7,105.4 . \mathrm{IR}(\mathrm{KBr}$, $\left.\mathrm{cm}^{-1}\right): 3134,1614,1555,1493,1469,1398,1315,1289,1229,1164,1010,886,859,765$, 728, 588. UV/Vis (acetonitrile): $\lambda_{\max }(\varepsilon)=265$ (1.234), 360 (0.986) nm. HPLC purity: 95.74\%. HRMS (ESI-Orbitrap) $m / z$ : $[\mathrm{M}+\mathrm{H}]^{+}$Calcd for $\mathrm{C}_{12} \mathrm{H}_{8} \mathrm{~N}_{2} \mathrm{O}_{3}+\mathrm{H}^{+}$229.06077; Found 229.06079.

Thiophen-2-yl-[4-(thiophen-2-yl)-1H-imidazol-2-yl]methanone (2o) and thiophen-2-yl-[5(thiophen-2-yl)-1H-imidazol-2-yl]methanone $\left(\mathbf{2 o}^{\prime}\right)$. Black solid. Mp: $228-230{ }^{\circ} \mathrm{C}$. NMR (500 MHz, DMSO-d $)_{6}$ ) $\delta 13.97(\mathrm{~s}, 0.2 \mathrm{H}), 13.69(\mathrm{~s}, 1 \mathrm{H}), 8.68(\mathrm{~s}, 1 \mathrm{H}), 8.59$ (s, 0.1H), 8.12 (d, J $5.0 \mathrm{~Hz}, 1.2 \mathrm{H}), 7.94(\mathrm{~s}, 1 \mathrm{H}), 7.73(\mathrm{~m}, 0.2 \mathrm{H}), 7.59(\mathrm{~m}, 0.4 \mathrm{H}), 7.48(\mathrm{~s}, 2 \mathrm{H}), 7.34$ (t, J $4.0 \mathrm{~Hz}$, 1.2H), $7.11(\mathrm{~s}, 1.2 \mathrm{H}) .{ }^{13} \mathrm{C}$ NMR $\left(125 \mathrm{MHz}, \mathrm{DMSO}-d_{6}\right): \delta 172.6,143.5,140.6,138.2,137.2$, $136.5,136.1,128.5,127.9,124.6,123.0,118.0 . \mathrm{IR}\left(\mathrm{KBr}, \mathrm{cm}^{-1}\right): 3235,2359,1603,1473$, $1413,1350,1274,1116,1051,824,777,734,692 . \mathrm{UV} / \mathrm{Vis}$ (acetonitrile): $\lambda_{\max }(\varepsilon)=198$ (1.072), 278 (1.278), 362 (1.071) nm. HPLC purity: 98.35\%. HRMS (ESI-Orbitrap) $m / z:[\mathrm{M}+$ $\mathrm{H}]^{+}$Calcd for $\mathrm{C}_{12} \mathrm{H}_{8} \mathrm{~N}_{2} \mathrm{OS}_{2}+\mathrm{H}^{+}$261.01508; Found 261.01511.

Thiazol-2-yl-[4-(thiazol-2-yl)-1H-imidazol-2-yl]methanone (2p) and thiazol-2-yl(5(thiazol-2-yl)-1H-imidazol-2-yl)methanone (2p'). Yellow solid. Mp: 338-342 ${ }^{\circ} \mathrm{C}$. NMR (500 MHz, DMSO-d $\left.)_{6}\right): \delta 14.08$ (s, 1H), 8.33 (d, J $\left.3.0 \mathrm{~Hz}, 1 \mathrm{H}\right), 8.28$ (d, J $\left.3.0 \mathrm{~Hz}, 1 \mathrm{H}\right), 8.09$ (s, $1 \mathrm{H}), 7.89(\mathrm{~d}, J 3.5 \mathrm{~Hz}, 1 \mathrm{H}), 7.74(\mathrm{~d}, J 3.5 \mathrm{~Hz}, 1 \mathrm{H}) .{ }^{13} \mathrm{C}$ NMR $\left(100 \mathrm{MHz}, \mathrm{DMSO}-d_{6}\right): \delta 170.3$, 162.0, 161.6, 145.0, 143.6, 143.3, 138.5, 129.2, 120.6, 119.8. IR (KBr, $\left.\mathrm{cm}^{-1}\right): 3701,3014$, 1662, 1467, 1396, 1359, 1268, 1136, 1111, 898, 879, 819, 738, 712. UV/Vis (acetonitrile): $\lambda_{\max }(\varepsilon)=195(0.823), 283$ (0.893), 360 (0.968) nm. HPLC purity: 98.31\%. HRMS (ESIOrbitrap) $m / z:[\mathrm{M}+\mathrm{H}]^{+}$Calcd for $\mathrm{C}_{10} \mathrm{H}_{6} \mathrm{~N}_{4} \mathrm{OS}_{2}+\mathrm{H}^{+}$263.00558; Found 263.00549.

Naphthalen-1-yl-[4-(naphthalen-1-yl)-1H-imidazol-2-yl]methanone (2q) and naphthalen1-yl-[5-(naphthalen-1-yl)-1H-imidazol-2-yl]methanone (2q'). Yellow solid. Mp: 174-180 ${ }^{\circ} \mathrm{C}$. NMR (400 MHz, DMSO- $\left.d_{6}\right): \delta 14.09(\mathrm{~s}, 0.2 \mathrm{H}), 13.97(\mathrm{~s}, 0.9 \mathrm{H}), 8.59(\mathrm{~d}, J \quad 8.4 \mathrm{~Hz}, 1 \mathrm{H})$, $8.32(\mathrm{~d}, J 7.6 \mathrm{~Hz}, 1.1 \mathrm{H}), 8.26(\mathrm{~d}, J 7.2 \mathrm{~Hz}, 1.1 \mathrm{H}), 8.18(\mathrm{~d}, J 8.0 \mathrm{~Hz}, 1.6 \mathrm{H}), 8.06$ (m, 1.6H), 
$8.03(\mathrm{~s}, 1.3 \mathrm{H}), 7.94(\mathrm{~d}, J 8.0 \mathrm{~Hz}, 1.1 \mathrm{H}), 7.89$ (d, J $8.0 \mathrm{~Hz}, 1 \mathrm{H}), 7.74(\mathrm{~d}, J 7.2 \mathrm{~Hz}, 1.4 \mathrm{H}), 7.69$ $(\mathrm{s}, 0.4 \mathrm{H}), 7.67(\mathrm{~s}, 0.9 \mathrm{H}), 7.62(\mathrm{~m}, 3.4 \mathrm{H}), 7.49(\mathrm{~m}, 3.6 \mathrm{H}) .{ }^{13} \mathrm{C}$ NMR $\left(100 \mathrm{MHz}, \mathrm{DMSO}-d_{6}\right): \delta$ $184.9,145.4,142.9,134.1,133.6,133.3,131.9,131.6,130.9,130.5,130.3,128.6,128.2$, $127.9,127.4,127.1,126.6,126.4,126.3,125.9,125.8,125.5,125.2,124.7,121.7 . \mathrm{IR}(\mathrm{KBr}$, $\left.\mathrm{cm}^{-1}\right): 3414,3261,1639,1366,1282,904,772,622 . \mathrm{UV} / \mathrm{Vis}$ (acetonitrile): $\lambda_{\max }(\varepsilon)=217$ (1.661), 330 (0.278) nm. HPLC purity: 95.42\%. HRMS (ESI-Orbitrap) $m / z:[\mathrm{M}+\mathrm{H}]^{+} \mathrm{Calcd}$ for $\mathrm{C}_{24} \mathrm{H}_{16} \mathrm{~N}_{2} \mathrm{O}+\mathrm{H}^{+} 349.13354$; Found 349.13354.

Naphthalen-2-yl-[4-(naphthalen-2-yl)-1H-imidazol-2-yl]methanone (2r) and naphthalen2-yl-[5-(naphthalen-2-yl)-1H-imidazol-2-yl]methanone (2r'). Yellow solid. Mp: 264-266 ${ }^{\circ} \mathrm{C}$. NMR (400 MHz, DMSO- $\left.d_{6}\right): \delta 13.98(\mathrm{~s}, 0.2 \mathrm{H}), 13.74(\mathrm{~s}, 1 \mathrm{H}), 9.44(\mathrm{~s}, 1 \mathrm{H}), 9.27(\mathrm{~s}, 0.3 \mathrm{H})$, $8.61(\mathrm{~s}, 0.3 \mathrm{H}), 8.51(\mathrm{~m}, 2.1 \mathrm{H}), 8.43(\mathrm{~s}, 0.3 \mathrm{H}), 8.24(\mathrm{~s}, 2.3 \mathrm{H}), 8.12(\mathrm{~m}, 3 \mathrm{H}), 8.04(\mathrm{~d}, J 8.0 \mathrm{~Hz}$, 1.5H), $7.98(\mathrm{~d}, J 8.0 \mathrm{~Hz}, 2.9 \mathrm{H}), 7.91(\mathrm{~d}, J 8.0 \mathrm{~Hz}, 1.6 \mathrm{H}), 7.68(\mathrm{~m}, 2.8 \mathrm{H}), 7.50(\mathrm{~m}, 2.8 \mathrm{H}) .{ }^{13} \mathrm{C}$ NMR (100 MHz, DMSO- $\left.d_{6}\right): \delta 180.7,145.1,143.0,135.8,135.0,133.3,133.3,133.1,132.4$, $132.0,131.2,130.1,128.8,128.2,128.0,127.9,127.7,126.9,126.4,125.9,125.7,123.8$, 122.9, 119.3. IR (KBr, cm $\left.{ }^{-1}\right): 3416,3282,1632,1612,1482,1448,1280,1161,781$. UV/Vis (acetonitrile): $\lambda_{\max }(\varepsilon)=215$ (2.404), 256 (1.652), 355 (0.568) nm. HPLC purity: 95.05\%. HRMS (ESI-Orbitrap) $m / z$ : $[\mathrm{M}+\mathrm{H}]^{+}$Calcd for $\mathrm{C}_{24} \mathrm{H}_{16} \mathrm{~N}_{2} \mathrm{O}+\mathrm{H}^{+} 349.13354$; Found 349.13333.

Quinolin-3-yl-[4-(quinolin-3-yl)-1H-imidazol-2-yl]methanone (2s) and quinolin-3-yl-[5(quinolin-3-yl)-1H-imidazol-2-yl]methanone $\left(\mathbf{2 s}^{\prime}\right)$. Yellow solid. Mp: $268-273{ }^{\circ} \mathrm{C}$. NMR (400 MHz, DMSO-d $)$ ): $\delta 14.27(\mathrm{~s}, 0.1 \mathrm{H}), 13.98(\mathrm{~s}, 1 \mathrm{H}), 9.84(\mathrm{~d}, J 2.0 \mathrm{~Hz}, 0.7 \mathrm{H}), 9.72$ (s, $0.1 \mathrm{H}), 9.66(\mathrm{~d}, J 1.6 \mathrm{~Hz}, 0.9 \mathrm{H}), 9.59(\mathrm{~d}, J 2.0 \mathrm{~Hz}, 0.1 \mathrm{H}), 9.52(\mathrm{~d}, J 2.0 \mathrm{~Hz}, 1 \mathrm{H}), 9.47$ (s, $0.2 \mathrm{H}), 8.96(\mathrm{~s}, 0.1 \mathrm{H}), 8.82(\mathrm{~d}, J 1.2 \mathrm{~Hz}, 1 \mathrm{H}), 8.42(\mathrm{~s}, 1 \mathrm{H}), 8.33(\mathrm{~d}, J 8.0 \mathrm{~Hz}, 1 \mathrm{H}), 8.22(\mathrm{~s}$, $0.2 \mathrm{H}), 8.14(\mathrm{~d}, J \quad 8.4 \mathrm{~Hz}, 1.2 \mathrm{H}), 8.04(\mathrm{dd}, J 13.2 \mathrm{~Hz}, J \quad 8.0 \mathrm{~Hz}, 2.3 \mathrm{H}), 7.95$ (m, 1.3H), 7.73 $(\mathrm{m}, 2.4 \mathrm{H}), 7.61(\mathrm{~m}, 1.3 \mathrm{H}) .{ }^{13} \mathrm{C}$ NMR $\left(100 \mathrm{MHz}, \mathrm{DMSO}-d_{6}\right): \delta 179.6,150.6,148.9,148.6$, $146.9,145.1,140.5,140.3,132.4,130.3,130.2$, 129.2, 128.8, 128.8, 128.7, 128.3, 127.8, 127.6, 127.1, 126.7, 126.5, 120.5. IR (KBr, cm $\left.{ }^{-1}\right): 3417,1636,1395,1326,1123,793,746$. UV/Vis (acetonitrile): $\lambda_{\max }(\varepsilon)=213(1.287), 254(1.075), 340(0.462) \mathrm{nm}$. HPLC purity: 95.60\%. HRMS (ESI-Orbitrap) $m / z$ : $[\mathrm{M}+\mathrm{H}]^{+}$Calcd for $\mathrm{C}_{22} \mathrm{H}_{14} \mathrm{~N}_{4} \mathrm{O}+\mathrm{H}^{+}$351.12404; Found 351.12405 .

[1]Benzofuran-2-yl-[4-([1]benzofuran-2-yl)-1H-imidazol-2-yl]methanone $\quad(2 \mathrm{u}) \quad$ and [1]benzofuran-2-yl-[5-([1]benzofuran-2-yl)-1H-imidazol-2-yl]methanone $\left(\mathbf{2} \mathbf{u}^{\prime}\right)$. Orange solid. Mp: 272-277 ${ }^{\circ} \mathrm{C}$. NMR (400 MHz, DMSO-d $\left.d_{6}\right): \delta 14.38$ (s, 0.2H), $14.02(\mathrm{~s}, 1 \mathrm{H}), 8.83$ (s, $0.6 \mathrm{H}), 8.68(\mathrm{~s}, 0.1 \mathrm{H}), 8.06(\mathrm{~s}, 0.7 \mathrm{H}), 7.99(\mathrm{~d}, J 5.2 \mathrm{~Hz}, 0.8 \mathrm{H}), 7.95(\mathrm{~s}, 0.2 \mathrm{H}), 7.80(\mathrm{~s}, 0.1 \mathrm{H})$, $7.77(\mathrm{~d}, J 5.6 \mathrm{~Hz}, 0.9 \mathrm{H}), 7.68(\mathrm{~d}, J 4.8 \mathrm{~Hz}, 1 \mathrm{H}), 7.62(\mathrm{~d}, J 5.6 \mathrm{~Hz}, 0.9 \mathrm{H}), 7.59$ (t, J 4.8 Hz, $1.1 \mathrm{H}), 7.54(\mathrm{~s}, 0.1 \mathrm{H}), 7.42(\mathrm{t}, J 4.8 \mathrm{~Hz}, 1.1 \mathrm{H}), 7.35(\mathrm{~s}, 0.8 \mathrm{H}), 7.32(\mathrm{t}, J 5.2 \mathrm{~Hz}, 0.9 \mathrm{H}), 7.27(\mathrm{t}$, $J$ 5.2 Hz, 1.1H). ${ }^{13} \mathrm{C}$ NMR (100 MHz, DMSO- $\left.d_{6}\right): \delta 169.8,155.3,154.1,151.2,150.2,144.4$, $134.8,128.9,128.7,127.4,127.1,124.3,124.2,123.5,123.3,121.1,120.3,119.4,112.2$, 111.1, 101.6. IR (KBr, cm $\left.{ }^{-1}\right): 3418,3248,1618,1548,1386,1348,1124,1027,726$. UV/Vis (acetonitrile): $\lambda_{\max }(\varepsilon)=197$ (1.184), 290 (0.673), 375 (0.455) nm. HPLC purity: 99.24\%. 
HRMS (ESI-Orbitrap) $m / z:[\mathrm{M}+\mathrm{H}]^{+}$Calcd for $\mathrm{C}_{20} \mathrm{H}_{12} \mathrm{~N}_{2} \mathrm{O}_{3}+\mathrm{H}^{+}$329.09207; Found 329.09207.

[1]Benzothiophen-2-yl-[(4-([1]benzothiophen-2-yl)-1H-imidazol-2-yl]methanone (2v) and [1]benzothiophen-2-yl-[5-([1]benzo[b]thiophen-2-yl)-1H-imidazol-2-yl]methanone $\left(2 \mathrm{v}^{\prime}\right)$. Yellow solid. Mp: 304-308 ${ }^{\circ} \mathrm{C}$. NMR (400 MHz, DMSO- $\left.d_{6}\right): \delta 14.32$ (s, 0.1H), 13.98 (s, 1H), $9.13(\mathrm{~s}, 0.8 \mathrm{H}), 9.10(\mathrm{~s}, 0.1 \mathrm{H}), 8.19(\mathrm{~m}, 2 \mathrm{H}), 8.14(\mathrm{~d}, J 8.0 \mathrm{~Hz}, 1 \mathrm{H}), 8.10(\mathrm{~s}, 0.1 \mathrm{H}), 8.01(\mathrm{~d}, J$ $7.6 \mathrm{~Hz}, 1 \mathrm{H}), 7.87(\mathrm{~m}, 2 \mathrm{H}), 7.76(\mathrm{~s}, 0.1 \mathrm{H}), 7.59(\mathrm{t}, J 8.0 \mathrm{~Hz}, 1.1 \mathrm{H}), 7.53(\mathrm{t}, J 7.6 \mathrm{~Hz}, 1.1 \mathrm{H})$, $7.38(\mathrm{~m}, 2.2 \mathrm{H}) .{ }^{13} \mathrm{C}$ NMR $\left(100 \mathrm{MHz}, \mathrm{DMSO}-d_{6}\right): \delta 173.8,143.9,142.5,140.4,140.3,138.9$, $138.5,138.2$, 137.3, 133.7, 127.9, 126.8, 125.3, 124.7, 124.3, 123.4, 123.0, 122.5, 120.0, 119.1. IR (KBr, cm $\left.{ }^{-1}\right): 3433,3241,2920,1607,1589,1513,1466,1243,1128,826,741$. UV/Vis (acetonitrile): $\lambda_{\max }(\varepsilon)=274(0.552), 352(0.360) \mathrm{nm}$. HPLC purity: 95.29\%. HRMS (ESI-Orbitrap) $m / z:[\mathrm{M}+\mathrm{H}]^{+}$Calcd for $\mathrm{C}_{20} \mathrm{H}_{12} \mathrm{~N}_{2} \mathrm{OS}_{2}+\mathrm{H}^{+} 361.04638$; Found 361.04590 .

[1]Benzothiophen-3-yl-[4-([1]benzothiophen-3-yl)-1H-imidazol-2-yl]methanone (2w) and [1]benzothiophen-3-yl-[(5-([1]benzothiophen-3-yl)-1H-imidazol-2-yl]methanone

$\left(\mathbf{2 w}^{\prime}\right)$,.Yellow solid. Mp: 229-233 ${ }^{\circ} \mathrm{C}$. NMR (400 MHz, DMSO- $\left.d_{6}\right): \delta 13.88(\mathrm{~s}, 0.2 \mathrm{H}), 13.73$ (s, $1 \mathrm{H}), 10.00(\mathrm{~s}, 0.8 \mathrm{H}), 9.83(\mathrm{~s}, 0.1 \mathrm{H}), 8.74(\mathrm{~d}, J 8.0 \mathrm{~Hz}, 1.1 \mathrm{H}), 8.56(\mathrm{~d}, J 8.0 \mathrm{~Hz}, 1 \mathrm{H}), 8.29$ $(\mathrm{s}, 0.2 \mathrm{H}), 8.18(\mathrm{~s}, 0.9 \mathrm{H}), 8.12(\mathrm{~d}, J 8.0 \mathrm{~Hz}, 1.2 \mathrm{H}), 8.07(\mathrm{~s}, 1 \mathrm{H}), 8.03(\mathrm{~d}, J 8.0 \mathrm{~Hz}, 1 \mathrm{H}), 7.83(\mathrm{~s}$, $0.2 \mathrm{H}), 7.55(\mathrm{~m}, 1.2 \mathrm{H}), 7.48(\mathrm{~m}, 2.4 \mathrm{H}), 7.41(\mathrm{t}, J 7.6 \mathrm{~Hz}, 1.2 \mathrm{H}) .{ }^{13} \mathrm{C}$ NMR (100 MHz, DMSO$\left.d_{6}\right): \delta 175.6,144.8,143.4,140.0,139.1,138.6,137.4,136.7,131.6,129.7,125.8,125.4$, 124.8, 124.6, 124.5, 123.8, 123.7, 123.0, 119.1. IR (KBr, cm$\left.{ }^{-1}\right): 3468,3263,1608,1420$, 1395, 1111, 832, 742, 724. UV/Vis (acetonitrile): $\lambda_{\max }(\varepsilon)=194$ (1.123), 221 (1.604), 357 (0.484) nm. HPLC purity: 98.54\%. HRMS (ESI-Orbitrap) $m / z:[\mathrm{M}+\mathrm{H}]^{+}$Calcd for $\mathrm{C}_{20} \mathrm{H}_{12} \mathrm{~N}_{2} \mathrm{OS}_{2}+\mathrm{H}^{+} 361.04638$; Found 361.04639.

Phenanthren-9-yl-[4-(phenanthren-9-yl)-1H-imidazol-2-yl]methanone $\quad(2 x) \quad$ and phenanthren-9-yl-[5-(phenanthren-9-yl)-1H-imidazol-2-yl]methanone $\left(\mathbf{2} \mathbf{x}^{\prime}\right)$. White solid. Mp: 239-240 ${ }^{\circ} \mathrm{C}$. NMR (400 MHz, DMSO- $\left.d_{6}\right): \delta 14.23(\mathrm{~s}, 0.2 \mathrm{H}), 14.07(\mathrm{~s}, 1 \mathrm{H}), 8.95(\mathrm{~m}$, $1.8 \mathrm{H}), 8.90(\mathrm{~d}, J 8.4 \mathrm{~Hz}, 1.5 \mathrm{H}), 8.86(\mathrm{~d}, J 8.4 \mathrm{~Hz}, 1.1 \mathrm{H}), 8.80(\mathrm{~d}, J 8.0 \mathrm{~Hz}, 1 \mathrm{H}), 7.40(\mathrm{~d}, J 8.0$ $\mathrm{Hz}, 1 \mathrm{H}), 8.63(\mathrm{~s}, 1 \mathrm{H}), 8.54(\mathrm{~s}, 0.3 \mathrm{H}), 8.35$ (d, J 8.0 Hz, 1H), 8.31 (d, J 8.0 Hz, 0.2H), 8.18 (m, $1.6 \mathrm{H}), 8.14(\mathrm{~m}, 1.2 \mathrm{H}), 8.10(\mathrm{~s}, 0.2 \mathrm{H}), 8.07(\mathrm{~s}, 1.1 \mathrm{H}), 7.98(\mathrm{~d}, J 7.6 \mathrm{~Hz}, 1 \mathrm{H}), 7.73(\mathrm{~m}, 9.6 \mathrm{H})$, $7.56(\mathrm{t}, J 8.0 \mathrm{~Hz}, 1 \mathrm{H}) .{ }^{13} \mathrm{C} \mathrm{NMR}\left(100 \mathrm{MHz}, \mathrm{DMSO}-d_{6}\right): \delta 184.8,145.4,142.9,132.9,131.8$, $131.0,130.9,130.2,130.0,129.9,129.7,129.5,129.0,128.7,128.6,127.5,127.3,127.2$, $127.0,127.0,126.8,126.7,126.0,123.4,123.1,123.0,122.8,122.2 . \mathrm{IR}\left(\mathrm{KBr}, \mathrm{cm}^{-1}\right): 3432$, 1595, 1384, 1105, 742, 617. UV/Vis (acetonitrile): $\lambda_{\max }(\varepsilon)=208(0.258), 249(1.242) \mathrm{nm}$. HPLC purity: 95.91\%. HRMS (ESI-Orbitrap) $m / z$ : $[\mathrm{M}+\mathrm{H}]^{+}$Calcd for $\mathrm{C}_{32} \mathrm{H}_{20} \mathrm{~N}_{2} \mathrm{O}+\mathrm{H}^{+}$ 449.16484; Found 449.16412.

3,6-Diphenylpyrazin-2-ol (3a). Yellow solid. Mp: 339-342 ${ }^{\circ} \mathrm{C}$. NMR (400 MHz, DMSO- $d_{6}$ ): $\delta 12.46(\mathrm{~s}, 0.9 \mathrm{H}), 8.29(\mathrm{~d}, J 6.8 \mathrm{~Hz}, 2 \mathrm{H}), 7.92(\mathrm{~s}, 2 \mathrm{H}), 7.53(\mathrm{~m}, 3 \mathrm{H}), 7.46(\mathrm{~m}, 3 \mathrm{H}) .{ }^{13} \mathrm{C} \mathrm{NMR}$ $\left(100 \mathrm{MHz}, \mathrm{DMSO}-d_{6}\right): \delta 156.2,136.0,130.1,129.3,129.0,128.4,128.0,127.0 . \mathrm{IR}\left(\mathrm{KBr}, \mathrm{cm}^{-}\right.$ $\left.{ }^{1}\right): 3533,3058,2910,2868,1637,1248,768,752,690,579$. UV/Vis (acetonitrile): $\lambda_{\max }(\varepsilon)=$ 
240 (0.291), 352 (0.499) nm. HPLC purity: 99.36\%. HRMS (ESI-Orbitrap) $m / z:[\mathrm{M}+\mathrm{H}]^{+}$ Calcd for $\mathrm{C}_{16} \mathrm{H}_{12} \mathrm{~N}_{2} \mathrm{O}+\mathrm{H}^{+}$249.10224; Found 249.10222.

3,6-Bis(3-chlorophenyl)pyrazin-2-ol (3c). Yellow solid. Mp: 297-301 ${ }^{\circ} \mathrm{C}$. NMR (400 MHz, DMSO-d $\left.d_{6}\right): \delta 12.58(\mathrm{~s}, 0.6 \mathrm{H}), 8.37(\mathrm{~m}, 1 \mathrm{H}), 8.26(\mathrm{t}, J 3.2 \mathrm{~Hz}, 1.1 \mathrm{H}), 8.06(\mathrm{~s}, 1 \mathrm{H}), 7.95(\mathrm{~s}$, $1.1 \mathrm{H}), 7.58(\mathrm{~m}, 2 \mathrm{H}), 7.52(\mathrm{~m}, 2.2 \mathrm{H}) .{ }^{13} \mathrm{C}$ NMR (100 MHz, DMSO- $\left.d_{6}\right): \delta$ 156.3, 137.8, 133.9, $132.9,130.9,130.1,129.9,129.1,128.0,127.0,126.8,125.7 . \mathrm{IR}\left(\mathrm{KBr}, \mathrm{cm}^{-1}\right): 3417,2923$, 1651, 1261, 1086, 1018, 792. UV/Vis (acetonitrile): $\lambda_{\max }(\varepsilon)=238(0.292), 338(0.397) \mathrm{nm}$. HPLC purity: $97.85 \%$. HRMS (ESI-Orbitrap) $m / z$ : $[\mathrm{M}+\mathrm{H}]^{+}$Calcd for $\mathrm{C}_{16} \mathrm{H}_{10} \mathrm{Cl}_{2} \mathrm{~N}_{2} \mathrm{O}+\mathrm{H}^{+}$ 317.02429; Found 317.02411.

3,6-Di(pyridin-2-yl)pyrazin-2-ol (3j). Orange solid. Mp: 247-248 ${ }^{\circ} \mathrm{C}$. NMR (500 MHz, DMSO- $\left.d_{6}\right): \delta 15.36(\mathrm{~s}, 1 \mathrm{H}), 9.21(\mathrm{~s}, 0.8 \mathrm{H}), 8.78(\mathrm{ddd}, J 5.0 \mathrm{~Hz}, J 1.5 \mathrm{~Hz}, J 0.5 \mathrm{~Hz}, 1 \mathrm{H}), 8.76$ (ddd, J 5.0 Hz, J 2.0 Hz, J 1.0 Hz, 1H), 8.60 (d, J $3.0 \mathrm{~Hz}, 1 \mathrm{H}), 8.33$ (d, J 8.0 Hz, 1H), 8.20 $(\mathrm{td}, J 8.0 \mathrm{~Hz}, J 1.5 \mathrm{~Hz}, 1 \mathrm{H}), 8.02(\mathrm{td}, J 7.5 \mathrm{~Hz}, J 1.5 \mathrm{~Hz}, 1 \mathrm{H}), 7.69(\mathrm{td}, J 6.0 \mathrm{~Hz}, J 0.5 \mathrm{~Hz}$, $1 \mathrm{H}), 7.54$ (ddd, $J 6.0 \mathrm{~Hz}, J 5.0 \mathrm{~Hz}, J 1.5 \mathrm{~Hz}, 1 \mathrm{H}) .{ }^{13} \mathrm{C}$ NMR $\left(120 \mathrm{MHz}, \mathrm{DMSO}-d_{6}\right): \delta 159.8$, $154.3,153.1,149.6,149.2,146.3,139.5,137.7,133.1,133.1,125.1,125.0,121.5,121.2$. IR $\left(\mathrm{KBr}, \mathrm{cm}^{-1}\right): 3432,2922,2358,1596,1362,1105,789 . \mathrm{UV} / \mathrm{Vis}$ (acetonitrile): $\lambda_{\max }(\varepsilon)=195$ (0.904), 251 (0.309), 353 (0.785) nm. HPLC purity: 98.33\%. HRMS (ESI-Orbitrap) $m / z:[\mathrm{M}+$ $\mathrm{H}]^{+}$Calcd for $\mathrm{C}_{14} \mathrm{H}_{10} \mathrm{~N}_{4} \mathrm{O}+\mathrm{H}^{+} 251.09274$; Found 251.09279.

3,6-Di(pyridin-3-yl)pyrazin-2-ol (3k). Yellow solid. Mp: 284-287 ${ }^{\circ} \mathrm{C}$. NMR $(500 \mathrm{MHz}$, DMSO- $\left.d_{6}\right): \delta 9.74(\mathrm{dd}, J 2.0 \mathrm{~Hz}, J 0.5 \mathrm{~Hz}, 0.9 \mathrm{H}), 9.23(\mathrm{~d}, J 1.5 \mathrm{~Hz}, 1 \mathrm{H}), 8.90$ (dt, $J 8.0 \mathrm{~Hz}, J$ $2.0 \mathrm{~Hz}, 1 \mathrm{H}), 8.55$ (dd, J $5.0 \mathrm{~Hz}, J 1.5 \mathrm{~Hz}, 1 \mathrm{H}), 8.43$ (dd, J $5.0 \mathrm{~Hz}, J 2.0 \mathrm{~Hz}, 1 \mathrm{H}), 8.38$ (dt, $J$ $8.0 \mathrm{~Hz}, J 1.5 \mathrm{~Hz}, 1 \mathrm{H}), 8.03$ (s, 1H), 7.43 (dd, J 7.5 Hz, J 4.5 Hz, 1H), 7.35 (ddd, J 8.0 Hz, J $4.5 \mathrm{~Hz}, J \quad 0.5 \mathrm{~Hz}, 1 \mathrm{H}) .{ }^{13} \mathrm{C}$ NMR $\left(125 \mathrm{MHz}, \mathrm{DMSO}-d_{6}\right): \delta$ 166.0, 149.0, 148.9, 147.7, 147.2, $146.9,138.2,135.0,134.4,133.6,133.6,123.4,122.5,121.9 . \mathrm{IR}\left(\mathrm{KBr}, \mathrm{cm}^{-1}\right): 3435,3049$, 2919, 1963, 1652, 1553, 1511, 1428, 1412, 1308, 1101, 1010, 812, 700. UV/Vis (acetonitrile): $\lambda_{\max }(\varepsilon)=260$ (0.168), 403 (0.101) nm. HPLC purity: 95.29\%. HRMS (ESI-Orbitrap) $m / z$ : [M $+\mathrm{H}]^{+}$Calcd for $\mathrm{C}_{14} \mathrm{H}_{10} \mathrm{~N}_{4} \mathrm{O}+\mathrm{H}^{+} 251.09274$; Found 251.09273 .

3,6-Di(pyridin-4-yl)pyrazin-2-ol (3l). Orange solid. Mp: 316-324 ${ }^{\circ} \mathrm{C}$. NMR $(400 \mathrm{MHz}$, DMSO-d $)_{6}: \delta 8.74(\mathrm{dd}, J 4.8 \mathrm{~Hz}, J 1.6 \mathrm{~Hz}, 2 \mathrm{H}), 8.69$ (dd, $\left.J 4.8 \mathrm{~Hz}, J 1.2 \mathrm{~Hz}, 2 \mathrm{H}\right), 8.61$ (s, 1H), 8.19 (dd, $J 4.8 \mathrm{~Hz}, J 1.2 \mathrm{~Hz}, 2 \mathrm{H}), 7.98$ (d, $J 6.0 \mathrm{~Hz}, 2 \mathrm{H}) .{ }^{13} \mathrm{C}$ NMR (100 MHz, DMSO$\left.d_{6}\right): \delta 157.0,150.6,150.5,150.2,149.9,149.8,142.7,122.8,122.5,122.5,122.4,121.4$, 121.1, 120.9. IR (KBr, cm $\left.{ }^{-1}\right): 3420,3059,2916,1653,1417,1310,1248,1080,929,826,781$. UV/Vis (acetonitrile): $\lambda_{\max }(\varepsilon)=195$ (1.443), 248 (0.540), 343 (0.727) nm. HPLC purity: 99.46\%. HRMS (ESI-Orbitrap) $m / z$ : $[\mathrm{M}+\mathrm{H}]^{+}$Calcd for $\mathrm{C}_{14} \mathrm{H}_{10} \mathrm{~N}_{4} \mathrm{O}+\mathrm{H}^{+}$251.09274; Found 251.09274.

3,6-Di(thiazol-2-yl)pyrazin-2-ol (3p). Yellow solid. Mp: 276-280 ${ }^{\circ} \mathrm{C}$. NMR (400 MHz, DMSO-d $\left.)_{6}\right): \delta .93(\mathrm{~s}, 0.8 \mathrm{H}), 8.15(\mathrm{~d}, J 3.2 \mathrm{~Hz}, 1 \mathrm{H}), 8.11(\mathrm{~d}, J 2.8 \mathrm{~Hz}, 1 \mathrm{H}), 8.06(\mathrm{~d}, J 3.2 \mathrm{~Hz}$, $1 \mathrm{H}), 8.03(\mathrm{~d}, J 3.2 \mathrm{~Hz}, 1 \mathrm{H}) .{ }^{13} \mathrm{C}$ NMR $\left(100 \mathrm{MHz}, \mathrm{DMSO}-d_{6}\right): \delta 156.7,145.1,143.0,129.7$, 124.3, 123.8. IR (KBr, cm $\left.{ }^{-1}\right): 3348,3076,2921,2426,1868,1516,1466,1418,1402,1227$, 
1191, 1079, 1050, 990, 763. UV/Vis (acetonitrile): $\lambda_{\max }(\varepsilon)=194(0.439), 290$ (0.162), 377

(0.772) nm. HPLC purity: 97.92\%. HRMS (ESI-Orbitrap) $m / z:[\mathrm{M}+\mathrm{H}]^{+}$Calcd for $\mathrm{C}_{10} \mathrm{H}_{6} \mathrm{~N}_{4} \mathrm{OS}_{2}+\mathrm{H}^{+}$263.00558; Found 263.00558.

\section{References and Notes}

1. Grimmett, M. R. Comprehensive Heterocyclic Chemistry II, Katritzky, A. R.; Rees, C. W.; Scriven, E. F. V., Eds.; Elsevier: Oxford, 1996; Vol. 3, p 77.

http://dx.doi.org/10.1016/B978-008096518-5.00060-5

2. Faulkner, D. J. Nat. Prod. Rep. 2000, 17, 7.

http://dx.doi.org/10.1039/a809395d

PMid:10714898

1. Bartik, K.; Braekman, J. C.; Daloze, D.; Stoller, C.; Huysecom, J.; Vandevyver, G.; Ottinger, R. Can. J. Chem. 1987, 65, 2118.

http://dx.doi.org/10.1139/v87-352

2. Oh, K. B.; Mar, W.; Kim, S.; Kim, J. Y.; Lee, T. H.; Kim, J. G.; Shin, D.; Sim, C. J.; Shin, J. Biol. Pharm. Bull. 2006, 29, 570.

http://dx.doi.org/10.1248/bpb.29.570

3. Duran, R.; Zubia, E.; Ortega, M. J.; Naranjo, S.; Salva, J. Tetrahedron 1999, 55, 13225. http://dx.doi.org/10.1016/S0040-4020(99)00803-0

4. Vlassara, H.; Valinsky, J.; Brownlee, M.; Cerami, C.; Nishimoto, S.; Cerami, A. J Exp Med 1987, 166, 539.

http://dx.doi.org/10.1084/jem.166.2.539

PMid:3598465

7. Chang, J. C. F.; Ulrich, P. C.; Bucala, R.; Cerami, A. J. Biol. Chem. 1985, 260, 7970.

8. Pongor, S.; Ulrich, P. C.; Bencsath, F. A.; Cerami, A. Proc. Natl. Acad. Sci. U. S. A. 1984, $81,2684$.

http://dx.doi.org/10.1073/pnas.81.9.2684

PMid:6585821 PMCid:PMC345134

9. Lapolla, A.; Gerhardinger, C.; Ghezzo, E.; Seraglia, R.; Sturaro, A.; Crepaldi, G.; Fedele, D.; Traldi, P. Biochim Biophys Acta 1990, 1033, 13.

http://dx.doi.org/10.1016/0304-4165(90)90187-2

10. Vlassara, H.; Brownlee, M.; Cerami, A. Proc. Natl. Acad. Sci. U. S. A. 1985, 82, 5588. PMid:2994035 PMCid:PMC390596

11. Nagaraj, M.; Boominathan, M.; Muthusubramanian, S.; Bhuvanesh, N. Synlett 2012, 23, 1353.

http://dx.doi.org/10.1055/s-0031-1290979 
12. Chen, J.; Chen, W.; Yu, Y.; Zhang, G. Tetrahedron Lett. 2013, 54, 1572. http://dx.doi.org/10.1016/j.tetlet.2013.01.042

13. Langhammer, I.; Erker, T. Heterocycles 2005, 65, 1975.

http://dx.doi.org/10.3987/COM-05-10445

14. Ueno, M.; Togo, H. Synthesis 2004, 2673.

15. Li, B.; Chiu, C. K. F.; Hank, R. F.; Murry, J.; Roth, J.; Tobiassen, H. Org. Process Res. Dev. 2002, 6, 682.

http://dx.doi.org/10.1021/op025552b

16. Heinrich, D. Justus Liebigs Annalen der Chemie 1858, 107, 199. http://dx.doi.org/10.1002/jlac.18581070209

17. Nicolaou, K. C.; Edmonds, D. J.; Bulger, P. G. Angew. Chem., Int. Ed. 2006, 45, 7134. http://dx.doi.org/10.1002/anie.200601872 PMid:17075967

18. Ihara, M. Arkivoc 2006, (vii), 416. http://dx.doi.org/10.3998/ark.5550190.0007.730

19. Chernyak, N.; Tilly, D.; Li, Z.; Gevorgyan, V. Arkivoc 2011, (v), 76.

20. Vanden Eynde, J. J.; Mayence, A. Arkivoc 2007, (iii), 96.

21. Cardwell, R. D.; Foreman, D. G.; Payne, T. R.; Wilbur, D. J. Arch. Environ. Contam. Toxicol. 1976, 4, 129.

http://dx.doi.org/10.1007/BF02221018

PMid:5060

22. Rietschel, L.; Langer, B. Berufsdermatosen 1965, 13, 111.

PMid:14336423

23. Lipinskii, S. Gig. Sanit. 1962, 27, 91.

PMid:14465689

24. Kornblum, N.; Powers, J. W.; Anderson, G. J.; Jones, W. J.; Larson, H. O.; Levand, O.; Weaver, W. M. J. Am. Chem. Soc. 1957, 79, 6562. http://dx.doi.org/10.1021/ja01581a057

25. Wan, Z.; Jones, C. D.; Mitchell, D.; Pu, J. Y.; Zhang, T. Y. J. Org. Chem. 2006, 71, 826. http://dx.doi.org/10.1021/jo051793g

PMid:16409004

26. Yusubov, M. S.; Filimonov, V. D.; Vasilyeva, V. P.; Chi, K. W. Synthesis 1995, 1234. http://dx.doi.org/10.1055/s-1995-4094

27. Cao, Z.; Liu, B.; Liu, W.; Yao, G.; Li, H.; Zou, T. J. Chem. Res. 2011, 35, 600. http://dx.doi.org/10.3184/174751911X13176543106368

28. Khalili, B.; Tondro, T.; Hashemi, M. M. Tetrahedron 2009, 65, 6882. http://dx.doi.org/10.1016/j.tet.2009.06.082 
29. Cao, L.; Ding, J.; Gao, M.; Wang, Z.; Li, J.; Wu, A. Org. Lett. 2009, 11, 3810. http://dx.doi.org/10.1021/ol901250c

PMid:19663463

30. Siemion, I. Z. Wiad. Chem. 1965, 19, 275.

31. The details of the crystal data have been deposited with Cambridge Crystallographic Data Centre as Supplementary Publication, CCDC 920522, 873930, 920523. 